\title{
Identification of a new stem cell population that generates Drosophila flight muscles
}

\author{
Rajesh D Gunage ${ }^{1}$, Heinrich Reichert², K VijayRaghavan ${ }^{1 *}$ \\ ${ }^{1}$ National Centre for Biological Sciences, Tata Institute of Fundamental Research, \\ Bangalore, India; ${ }^{2}$ Biozentrum, University of Basel, Basel, Switzerland
}

\begin{abstract}
How myoblast populations are regulated for the formation of muscles of different sizes is an essentially unanswered question. The large flight muscles of Drosophila develop from adult muscle progenitor (AMP) cells set-aside embryonically. The thoracic segments are all allotted the same small AMP number, while those associated with the wing-disc proliferate extensively to give rise to over 2500 myoblasts. An initial amplification occurs through symmetric divisions and is followed by a switch to asymmetric divisions in which the AMPs self-renew and generate postmitotic myoblasts. Notch signaling controls the initial amplification of AMPs, while the switch to asymmetric division additionally requires Wingless, which regulates Numb expression in the AMP lineage. In both cases, the epidermal tissue of the wing imaginal disc acts as a niche expressing the ligands Serrate and Wingless. The disc-associated AMPs are a novel muscle stem cell population that orchestrates the early phases of adult flight muscle development.

DOI: 10.7554/eLife.03126.001
\end{abstract}

*For correspondence: vijay@ ncbs.res.in

Competing interests: See page 21

Funding: See page 21

Received: 17 April 2014

Accepted: 15 August 2014

Published: 18 August 2014

Reviewing editor: Duojia Pan, Howard Hughes Medical Institute, Johns Hopkins University School of Medicine, United States

(c) Copyright Gunage et al. This article is distributed under the terms of the Creative Commons Attribution License, which permits unrestricted use and redistribution provided that the original author and source are credited.

\section{Introduction}

Stem cells populations can expand exponentially through symmetric division or asymmetrically divide to self-renew and produce a daughter cell, which differentiates to contribute to tissue formation or regeneration (Morrison and Kimble, 2006; Micchelli and Perrimon, 2006; Mandal et al., 2007; Maurange et al., 2008; Knoblich, 2001; Egger et al., 2008; Doe, 2008; Sousa-Nunes et al., 2010; Brand and Livesey, 2011). Many mature tissues such as colon, prostate, lung, muscle and brain use adult-specific stem cells in tissue maintenance or regeneration (Reya et al., 2001; Gonzalez, 2007). The molecular mechanisms of regulating stem-cell proliferation have been studied in neural-, intestinal-, hematopoietic- and epithelial-stem cells of vertebrates and invertebrates (Tulina and Matunis, 2001; Barker et al., 2007; Ohlstein and Spradling 2007; Takashima et al., 2008; Farkas and Hutter, 2008; Reichert, 2011; Homem and Knoblich 2012). The regulation of stemcell proliferation is usually through signals from a 'stem-cell niche' (Mandal et al., 2007; Brack et al., 2008; Chen et al., 2012; Cordero et al., 2012). Significant progress in understanding these mechanisms of regulation of stem-cell proliferation and self-renewal has been made in Drosophila. For example, in the developing optic lobe, stem cell-like neuroepithelial cells first increase in number through symmetric divisions and are then transformed into so-called neuroblasts which undergo self-renewing asymmetric divisions to generate differentiated neurons and glial cells in this part of the brain (Maurange et al., 2008; Egger et al., 2011). Recent work has identified signaling pathways and niches required for stem cell proliferation (Egger et al., 2010; Ngo et al., 2010; Reddy et al., 2010; Orihara-Ono et al., 2011; Wang and Rudnicki, 2011).

Drosophila flight muscles are formed from adult muscle precursors (AMPs) (Currie and Bate, 1991; Fernandes et al., 1991; Roy and VijayRaghavan, 1999). Myogenesis occurs in two phases; an embryonic one, which makes the muscles required for the larval life (Bate et al., 1991) while a postembryonic phase leads to formation of muscle required for the adult (Fernandes et al., 1991; Roy and VijayRaghavan, 1998; Sudarsan et al., 2001). The AMPs, lineal derivatives of the mesoderm, are generated embryonically and proliferate postembryonically (Bate et al., 1991; Fernandes et al., 
eLife digest Muscle tissues must grow and change to accommodate the needs of an animal at various stages in its life. For example, fruit flies begin life as larvae and their muscles must help them move their soft bodies. Later, when the flies mature into adults, the muscles must provide power for flight and support for the insects' external skeletons.

Like other animal tissues, muscles develop from non-specialized stem cells which at first have the potential to become almost any cell type, but later change to become more specialized. Studies of fruit flies, in particular, have yielded insights on how pools of stem cell are created and regulated. Fruit flies are small and easier to study than larger organisms, and as a result, scientists have learned a lot about their genetics and cell biology. Gunage et al. have now identified the stem cell pools that develop into flight muscle tissue, and found that these cells were set aside for the muscles when the fruit fly embryo was still developing.

Fruit flies have large forewings that power flight, and small modified hindwings (called halteres) that help the insect to balance when flying. Gunage et al. reveal that a small, but similar, number of cells are set aside to make both both the tiny muscles that will move the halteres and the much larger flight muscles that move the forewings. However, the cells that contribute to the flight muscles divide to give far more muscle progenitor cells than their haltere counterparts, and make a couple of thousand cells that eventually fuse to form muscle fibers.

Gunage et al. looked at how the flight muscle progenitors multiplied by genetically engineering some of the stem cells in fruit fly larvae so that when each cell divided, its two daughter cells would fluoresce with different colors. One daughter cell would glow green and the other glow red. Gunage et al. found that at first the cells multiply equally, with half the new cells coming from a 'red' stem cell and the other half from a 'green' cell-meaning that the number of cells increases exponentially. Later, the balance shifted so that either more red cells than green cells were produced, or vice versa. This results in a 'linear' increase in number of muscle progenitor cells. Furthermore, Gunage et al. identified the proteins that orchestrate the switch from equal to unequal multiplying of these cells at the different times points in the fruit flies' development.

The next challenge is to see if these stem cells that form the muscles are also available for repair of mature muscle tissue after it is damaged. If this is so, these stems cells might perform a similar function to muscle satellite cells, which are found in the mature muscles of mammals and other vertebrates.

DOI: 10.7554/eLife.03126.002

1991; Roy and VijayRaghavan, 1999). Little is known about the cellular and molecular mechanisms by which the AMPs proliferate and to give rise to the large number of cells which are needed to contribute to the massive adult flight muscles. During late embryogenesis the AMPs required for the formation of flight muscles are set aside in the mesothoracic segment (T2) and those required for haltere muscle development in the metathoracic segment (T3) (Sudarsan et al., 2001; Roy et al., 1997). The numbers of AMPs at this early stage in T2 and T3 are same but the AMPs in T2 proliferate profusely while those in T3 far less. Studies on the 'four-winged-fly' have clearly shown the key role played by the wing-disc ectoderm in regulating myoblast proliferation (Fernandes et al., 1994; Dutta et al., 2004; Roy and VijayRaghavan 1997). Yet, the mechanisms that regulate the amplification of muscle precursors to generate large 'pools of myoblasts', a feature common to adult muscles in the fly as well as to vertebrate skeletal muscles, (Sudarsan et al., 2001) have not been studied in the fly or indeed other systems.

In this report, we use clonal MARCM (Yu et al., 2009) techniques to study the proliferative activity of AMPs during postembryonic development. We focus on the AMPs associated with the wing imaginal disc in the second thoracic segment, which give rise to the large indirect flight muscles. We show that an initial amplification of the number of these AMPs occur through symmetric divisions and is followed by a switch to asymmetric divisions, in which the AMPs self-renew and generate postmitotic myoblasts required for the formation of adult myofibers. The sequential nature of these two division modes results in a change in the arrangement of AMP lineages from an initially monostratified layer adjacent to the wing disc epithelium to a markedly multistratified layer comprising both AMPs and their post mitotic myoblast progeny. While the initial amplification of AMPs through symmetric divisions 
is controlled by Notch signaling, the switch to the subsequent asymmetric division mode of AMP division additionally requires Wingless. In both cases the epidermal tissue of the wing imaginal disc acts as a stem cell niche and provides the ligands, Serrate and Wingless, for the two signaling pathways that operate in the AMPs. We identify the AMPs as a novel muscle stem cell population whose proliferation pattern orchestrates the building of the large flight muscles in Drosophila.

\section{Results}

\section{Wing disc associated AMPs proliferate during larval development to reach a population size of $\mathbf{2 5 0 0}$}

AMPs are lineal descendants of progenitor cells, derived from proneural gene-expressing equivalent groups in the embryonic mesoderm (Ruiz-Gómez and Bate, 1997; Carmena et al., 1998). In the thoracic segments of the embryo, each of these progenitor cells divides asymmetrically to generate a muscle founder cell involved in embryonic myogenesis and an AMP which has active Notch (N) signaling and continues to express the mesodermal marker Twist (Twi). In contrast to their muscle founder cell siblings, the AMPs do not differentiate in the embryo; rather they are set aside and at the end of embryonic development become associated with the imaginal discs (Bate et al., 1991). The Twi-expressing AMPs associated with the wing imaginal discs co-express Vestigial ( $\mathrm{Vg})$ in late embryonic stages (Sudarsan et al., 2001). In the early embryonic stage (Stage11) Wg induced DII (Distalless) expression leads to separation of the primordium for different discs and those for wing imaginal discs start expressing $\mathrm{Vg}$ and results in defining of the population for wing disc primordium (Cohen et al., 1993; Campos-Ortega, 1997; Kubota et al., 2000). To determine the number of AMPs present in the thoracic segments at the end of embryogenesis, we identified these cells using Twi-GAL4 > UAS-mCD8::GFP.

In each thoracic segment a total of approximately 10 cells were Twi-positive and dorsally located indicating that these correspond to the dorsal AMPs (Figure 1A) (Williams et al., 1991; Creig and Akam et al., 1993). To further characterize the extent of post-embryonic proliferation of the flightmuscle AMPs, we determined the number of Twi-positive cells associated with the T2 wing disc at different larval stages. Immediately after larval hatching (24 hr AEL) a total of $10( \pm 2)$ Twi-positive cells were found on the wing disc implying that these had not yet initiated proliferative divisions (Figure 1B). At the late second instar stage (72 hr AEL) the number of Twi-positive cells associated with the wing disc had increased to approximately $250( \pm 15)$ and at the late third instar stage (144 hr AEL) approximately $2500( \pm 90)$ Twi-positive cells were found on the wing disc indicative of intense proliferative activity (Figure 1C-E). We conclude that the $10 \mathrm{AMPs}$ present on the wing disc at the end of embryogenesis and the beginning of larval development initiate a proliferation process during subsequent larval stages which results in some 2500 Twi-positive cells at the end of larval development (Figure 1E,F,G). These findings suggest that each embryonically born AMP, on the average, would give rise to a lineage of approximately 250 cells during larval development. We next investigated the cellular mechanisms that make this remarkable proliferation possible.

\section{AMP proliferation involves initial symmetric and subsequent asymmetric clonal amplification}

To determine the mechanisms of AMP proliferation we used twin-spot MARCM, a genetic labeling technique which makes it possible to trace cell lineage and determine proliferation patterns by independently labeling the two paired sister cell siblings derived from a given cell division (Yu et al., 2009; O'Brien et al., 2011). In twin-spot MARCM, heat shock induces a timed mitotic recombination event in the precursor cell which results in differential labeling of its two daughter cell clones with heritable expression of green fluorescent protein (GFP) in one cell and red fluorescent protein (RFP) in the other. Symmetric cell divisions will result in daughter cell clones of RFP and GFP of equal number of cells; asymmetric clonal amplification should result in daughter cell clones of GFP and RFP of unequal size.

Twin-spot MARCM clones were induced in proliferating AMP lineages during larval development and recovered in the third instar. Clones were induced using the mesoderm-specific Dmef2-Gal4 driver (Ranganayakulu et al., 1998). Wing discs containing labeled clones were co-labeled for Twi-immunoreactivity. Twin-spot MARCM clones induced between the early first instar and the late second instar invariably resulted in two relatively large, differentially labeled daughter cell clones 


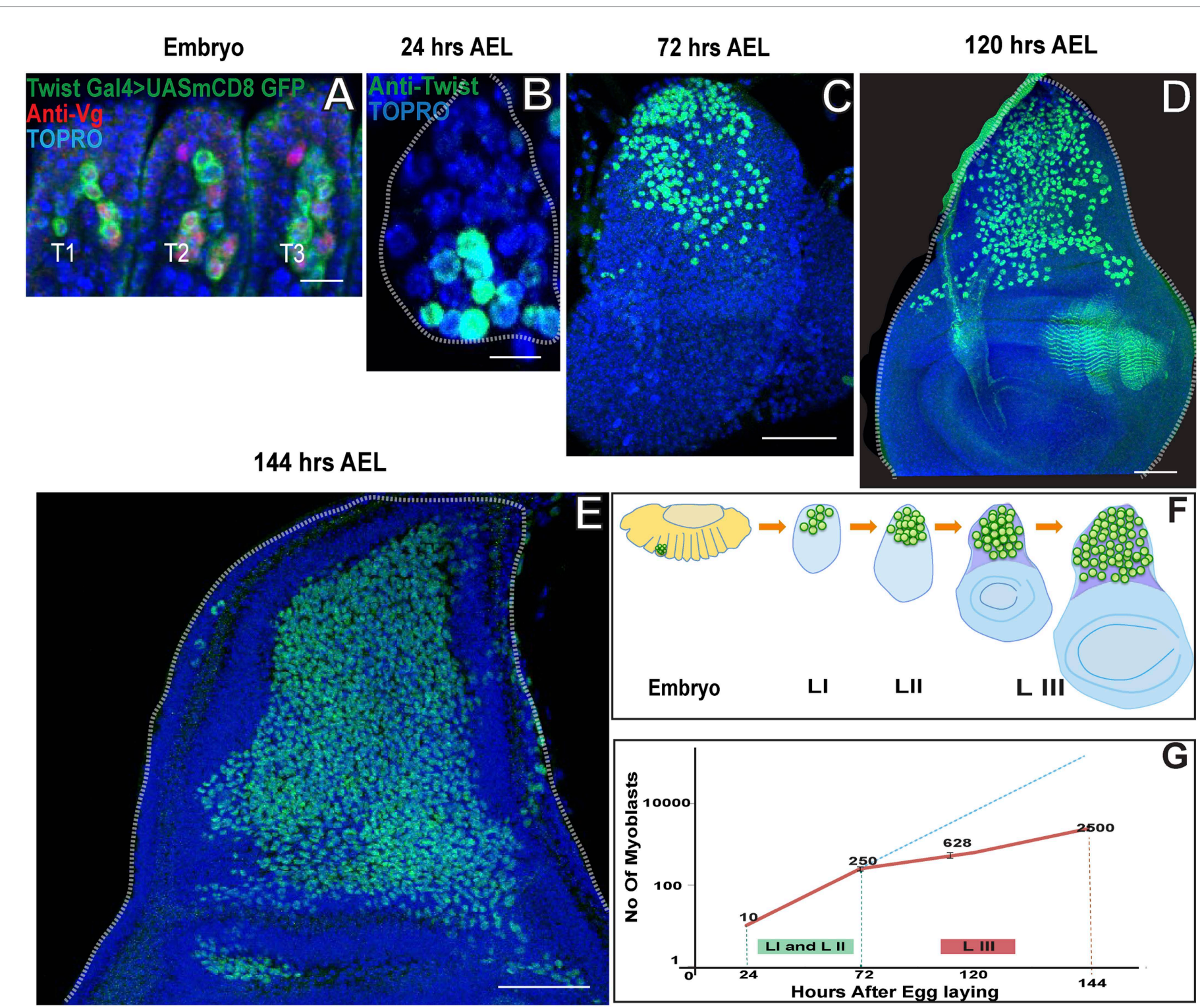

Figure 1. Wing disc associated AMPs proliferate during larval development to reach a population size of 2500 . (A) Stage 17 embryo ( 22 hr After Egg Laying (AEL) showing cluster of 10 AMPs in thoracic segment (T2) marked with Twist Gal4 > UAS mCD8GFP, Vg (anti-Vestigial, red) and TO-PRO3 (A nuclear stain, blue), Similar numbers of Twi positive cells are seen in each segment. $\mathrm{n}=5 \mathrm{Scale}$ bar, $10 \mu \mathrm{m}$. (B-E) Wing imaginal discs from early first ( $24 \mathrm{hr} A E L) n=5$. Scale bar, $10 \mu \mathrm{m}$, late second instar $(\sim 72 \mathrm{hr} A E L) n=10$ and third instar stage $(\sim 120 \mathrm{hr} A E L, n=10$ and $\sim 144 \mathrm{hr}$ $A E L, n=10$ ) stained for Twi (anti-Twist, green) and TO-PRO3 (A nuclear stain) showing increase in the number of AMPs during the larval instars. Scale bar, $50 \mu \mathrm{m}$. (F) Schematic showing AMPs, marked in green color, in T2 region of stage 17 embryo and subsequently in the presumptive notum of the first instar, second instar and late third instar wing imaginal disc. (G) A sharp increase is seen in the number of AMPs in first (I) and second (II) instars (Till $72 \mathrm{hr}$ AEL) (Depicted as red line). After $72 \mathrm{hr}$ AEL (Early third instar) till the end of third instar (144 hr AEL), the rate of increase of the AMP population is less sharp. The dotted blue line depicts the extrapolation of the early rate of growth. The graph depicts the average number of cells and the bar represents the standard error. For first instar ( $24 \mathrm{hr}$ ) $\mathrm{n}=5$, late second instar $(72 \mathrm{hr}$ ) $\mathrm{n}=10$, mid third instar $(120 \mathrm{hr}) \mathrm{n}=10$ and late third instar $(144 \mathrm{hr}) \mathrm{n}=10$

DOI: 10.7554/eLife.03126.003

that were similar in size and contained a similar number of cells (Figure 2A-C). This indicates that mitotically active cells in the AMP lineages divide symmetrically during early larval development (30 hr-72 hr AEL). In contrast, twin-spot MARCM clones induced during larval development from the early third instar ( $75 \mathrm{hr}$ AEL) onward always resulted in two differentially labeled daughter cell clones of different number of cells in each clone (Figure 2D-F). Notably, one of the two daughter 

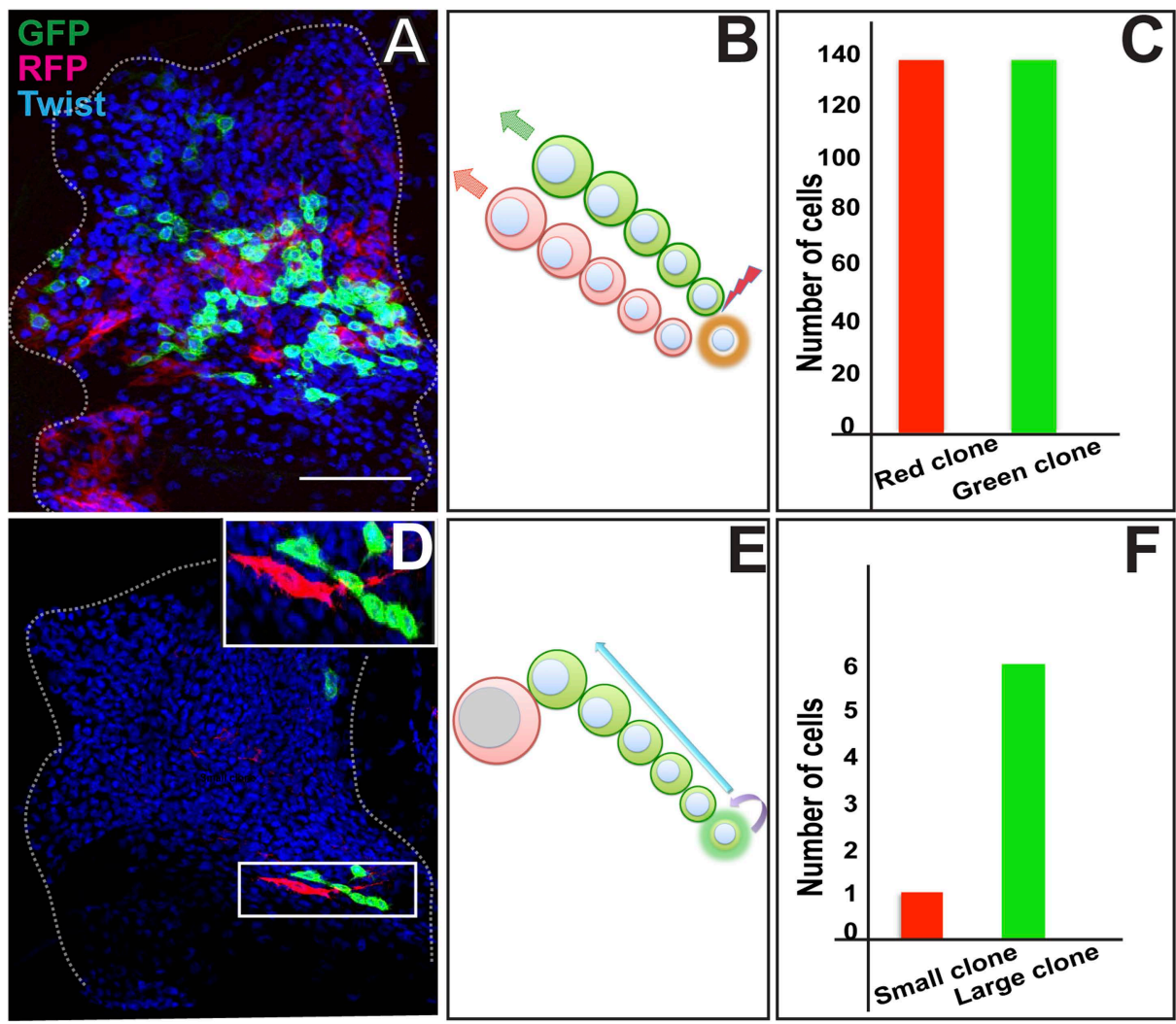

Figure 2. AMP proliferation involves initial symmetric and subsequent asymmetric clonal amplification. (A) Third instar wing imaginal disc ( 144 hr AEL) showing a Twin-spot MARCM clone induced by a single15 m heat shock at $37^{\circ} \mathrm{C}$ in the early first instar ( $24 \mathrm{hr}$ AEL). The GFP and RFP twin spots are of the same size. Anti-Twist marks all the descendants of AMP lineages. (B) Schematic depiction of clonal generation of symmetric clone from the AMP from the early first instar till late second instar stage. $N=10$ early clones were examined all with a similar result showing the twin spots of the same size. (C) Quantitation of the clone showing exactly same number (136) of cells in both red and green clone of twin-spot. (D) Asymmetric clone of AMP lineage recovered from a single $15 \mathrm{~m}$ heat shock at $37^{\circ} \mathrm{C}$ clonal induction in early third instar $(\sim 75 \mathrm{hr} \mathrm{AEL})$. Clone shows a single large red cell and six small green cells (zoomed image showed in top most right corner). Scale bar $50 \mu \mathrm{m}$. (E) Schematic depiction of clonal generation of asymmetric clone from the AMP in the early third instar onwards. $N=12$ late clones were examined all with a similar result. (F) Quantitation of clone showing one red and six green cells from the twin-spot marking experiment.

DOI: 10.7554/eLife.03126.004

cell clones invariably comprised one large labeled cell with elongated morphology, whereas the other daughter cell clone contained up to $10( \pm 3)$ smaller cells. This indicates that mitotically active cells in the AMP lineages have differential clonal potential and divide during the third larval instar stage (72 hr-144 hr AEL), with each stem cell giving rise to one stem cell through self-renewal as well as a sibling cell which could differentiate further.

Taken together, these findings indicate that proliferating cells in the AMP lineage divide in a symmetrical mode during early development such that 10 AMP progenitors in the early first larval instar stage give rise to a total of approximately 250 cells at the end of the second larval instar stage. In the third larval instar stage, these myogenic progenitor cells divide to produces up to 10 progeny to generate a total of approximately 2500 myogenic cells. This division is different from the earlier mode, as the division outcome is asymmetric as the majority of the clone is essentially due to proliferation of the one of the cell. Thus, by transiting through a sequence of symmetric cell divisions followed by asymmetric cell divisions, each embryonically generated AMP can generate a lineage of approximately 250 myogenic progeny during larval development. 


\section{Proliferating AMPs are located in a monolayer adjacent to the wing disc epithelium}

During the first and second larval instars, AMPs labeled with the Twi are located in a single monostratified layer adjacent to, and in contact with, the wing disc epithelium (Figure $3 A, B, B^{\prime}$ ). From the early third larval instar onward, labeled cells become organized in multistratified 2-3 cell layers on the wing disc (Figure $3 C_{1} C^{\prime}$; Video 1). To determine if mitotic activity takes place in specific layers, cells were co-labeled for phospo-histone-3 (PH3) immunoreactivity and Dmef2-Gal4 was used to drive UAS mCD8::GFP. Throughout larval development, PH3 positive cells were only observed in the cell layer immediately adjacent to the wing disc epithelium. Notably, even in the multistratified organization manifested in the third larval instar, $\mathrm{PH} 3$ positive cells were seen only in cells located adjacent to the epithelium and not in cells located in more distal layers (Figure 3D-D"). This implies that almost all mitotically active cells are located proximal to the disc epithelium, while the cells in the more distal layers are likely to be postmitotic. Taken together with the findings on cell division modes in the AMP lineages, these results also suggest that both symmetric and asymmetric cell divisions only take place in cells located adjacent to the wing disc epithelium.

Since twin-spot MARCM experiments indicate that a transition from a symmetrical to an asymmetrical mode of cell division occurs between the second and third larval instar stage, we focused on the mitotically active cells in the layer adjacent to the wing disc epithelium and examined the orientation of the axis of division at these two stages. Centrosome position and, hence, the axis of cell division were revealed by a GFP-tagged peri-centrosomal (CNN-GFP) label (Megraw et al., 2002) and Dmef2Gal4 was used to drive UAS-CNN-GFP expression. Throughout the second instar stage, the axis of cell division in the mitotically active cells is oriented parallel to the surface of the wing disc epithelium such that both daughter cells remain in contact with the epithelium (Figure 3E- $H^{\prime}$ ). In contrast, from early third instar stage onward, the axis of cell division shows an oblique to orthogonal orientation relative to the disc surface (Figure 3I-L'). Consequently, only one of the two daughter cells remains in contact with the disc epithelium while the other daughter cell loses epithelial contact and, hence, might contribute to the population of cells located in layers distal to the disc epithelium.

\section{Proliferating AMPs generate clones of postmitotic myoblasts localized in distal layers}

Given that proliferative mitotic activity in the AMP lineages only takes place in the cells located next to the epithelium we performed experiments to confirm that the cells in the more distal layers are indeed their postmitotic progeny. For this, we first performed EdU (5-Ethynyl-2'-deoxyuridine) incorporation experiments to visualize cells actively engaged in DNA replication as well as their progeny. EdU incorporation was carried out in early third instar larva (in a $5 \mathrm{hr}$ pulse) and visualized in AMP cells on wing discs co-labeled with for Twi immunoreactivity. When cells were examined directly after EdU incorporation (pulse, no chase), labeling was only found in cells adjacent to the disc epithelium (Figure $4 A, B$ ). This result confirms the finding that mitotically active cells are located next to the epithelium. If the incorporation of EdU was followed by $48 \mathrm{hr}$ without EdU (pulse, chase) and the cells then examined, labeling was seen in all layers. However, in this case, the majority of labeled cells were in the most distal layer, less labeled cells were in an intermediate layer and the lowest number of labeled cells was found in the layer adjacent to the disc (Figure $4 C_{,} D$ ). This indicates that the pulse of EdU labeling incorporated into the (replicating) cells adjacent to the disc epithelium was now manifest in the (non-replicating) cells located in more distal layers. This, in turn, implies that the cells in the more distal layers are the postmitotic progeny of the proliferating cells that remain in contact with the disc epidermis.

To further investigate the clonal nature of this proliferative process in the AMP lineages, we next carried out MARCM labeling experiments using the Dmef2-Gal4 driver. MARCM clones induced in the second larval stage and recovered in the third larval stage typically comprised 100-200 labeled cells arranged in a loose cluster and extending from the layer next to the disc epithelium to the most distal layer of cells (Figure 4E). Interestingly, within each clone the labeled cells in the distal layer were larger in size and had a more elongated morphology than those located in the innermost layer next to the disc (Figure 4G-N; Video 2). To determine if these clones comprised mitotically active cells, we combined MARCM labeling with PH3 immunolabeling. In all cases in which co-labeling was seen, only one cell within the clone was $\mathrm{PH} 3$-positive and this cell was always located adjacent to the disc epithelium (Figure $4 F_{\text {, }} F^{\prime}$; Video 3). 

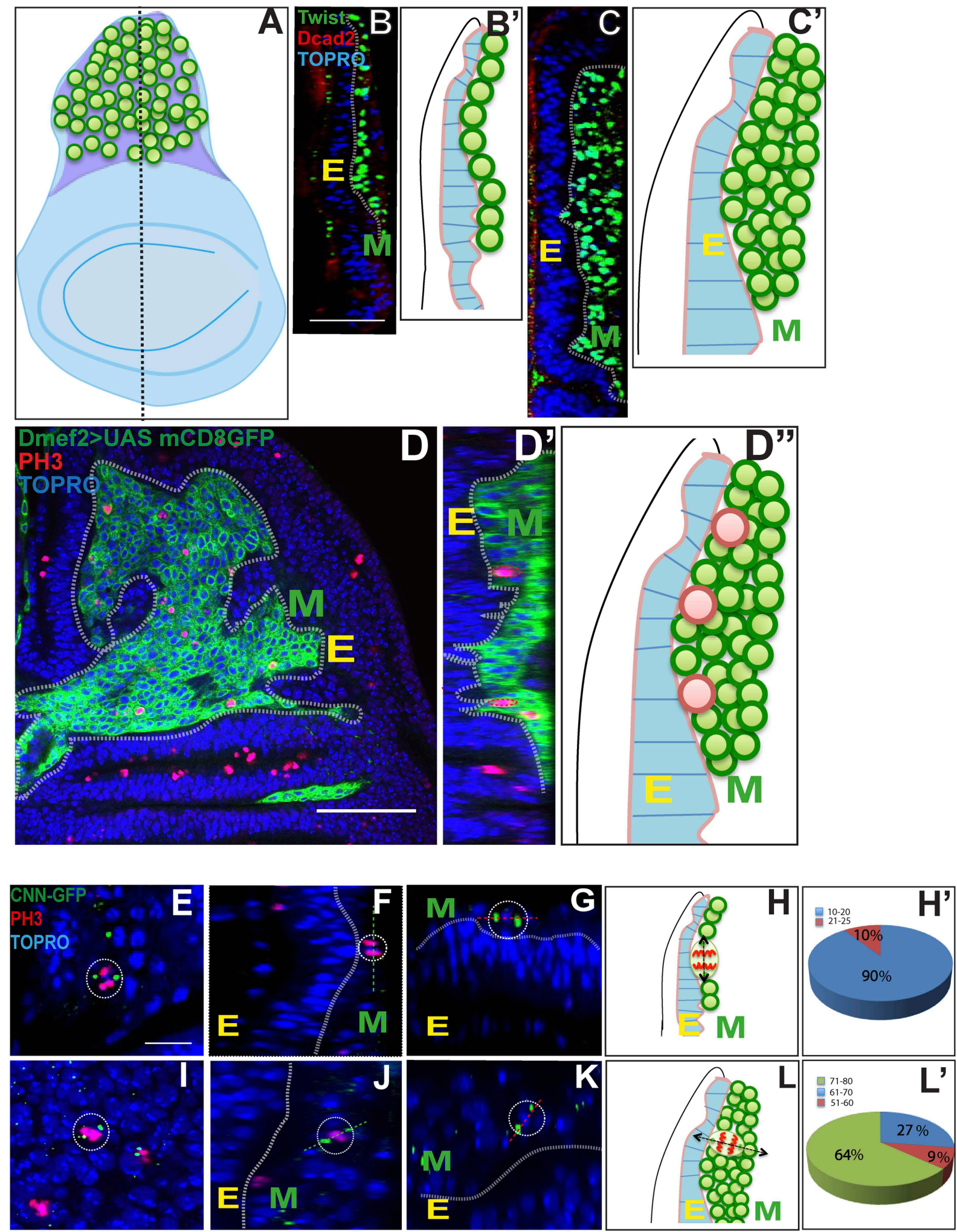

Figure 3. Proliferating AMPs are located in a monolayer adjacent to the wing disc epithelium. (A-B') Optical section of late second instar wing imaginal disc ( $72 \mathrm{hr}$ AEL, schematic in A with AMPs in green), showing monostratified (single layer) arrangement of AMPs (marked in green and denoted as M for mesoderm, B) stained for Twi (anti-Twist, green), Dcad2 (anti-Dcad2, red) (Disc-epithelium [E]) and TO-PRO3 (a nuclear stain, blue). $\mathrm{n}=10, \mathrm{Schematic}$ in $\mathbf{B}^{\prime}$. Figure 3. Continued on next page 
Figure 3. Continued

(C-C') Late third instar wing disc showing multistratified (3-4 layers) of AMPs in presumptive notum region. $\mathrm{n}=20$ Scale bar $50 \mu \mathrm{m}$. AMPs are stained for Twi (anti-Twi, green), epithelium (anti-Dcad2, red) and TOPRO (Nuclear stain, blue) (D-D") A late third instar wing imaginal disc showing multistratified arrangement of AMP using Dmef2-Gal4 > UASmCD8GFP (anti-GFP, green) and co-stained for mitotic marker PH-3 (anti-phosphohistone, red) and TO-PRO3 (Nuclear stain, blue). $\mathbf{C}^{\prime}$ shows a schematic representation. $\mathbf{D}^{\prime}$ shows an optical section of image $\mathbf{D}$ showing active mitotic divisions (red dotted circles) exclusively in the layer most-proximal to the disc epithelium (marked as $\mathbf{E}) . n=20$. $\mathbf{D}^{\prime \prime}$ represents this schematically. $(\mathbf{E}, \mathbf{F}$ and $\mathbf{G})$ Late second instar wing discs ( 70 hr AEL) marked for centrosomin-GFP (CNN-GFP, a pericentrosomal marker, green) using Dmef2-Gal4, PH3 (anti-phosphohistone, red) and TO-PRO3 (Nuclear stain, blue). In each panel, E is the Epidermis and M the AMPs. ( $\left.\mathbf{H}-\mathbf{H}^{\prime}\right)$ Schematic (H) of a late second instar disc (as shown in $\mathbf{F}$ ) showing active mitotic division (Black arrow) parallel to the disc epithelium. Pie-chart representation ( $\left.\mathrm{H}^{\prime}\right)\left(\mathrm{Blue}\right.$ : $10^{\circ}-20^{\circ}$ with $0^{\circ}$ being parallel to the epidermis. $\mathrm{n}=6$ preps. (I, J and $\mathbf{K})$ Third instar wing imaginal disc ( 140 hr AEL) showing a more orthogonal orientation in AMP marked using centrosomin-GFP (CNN-GFP) (a centrosome marker, green) using Dmef2-Gal4, PH3 (anti-phosphohistone, red) and TO-PRO3 (Nuclear stain, blue). $\mathrm{n}=10$. Scale bar, $10 \mu \mathrm{m}$. $\left(\mathbf{L}-\mathbf{L}^{\prime}\right)$ A schematic representation $(\mathbf{L})$ and pie-chart representation $\left(\mathbf{L}^{\prime}\right)$ of late third instar disc showing mitotic division axis (black arrow, in $\left.\mathbf{L}\right)$ of $A M P$ (green) oblique to disc surface (blue). The angle of division at this stage is in the range of $50-80^{\circ}$ as represented in $\mathbf{L}^{\prime}$ DOI: 10.7554/eLife.03126.005

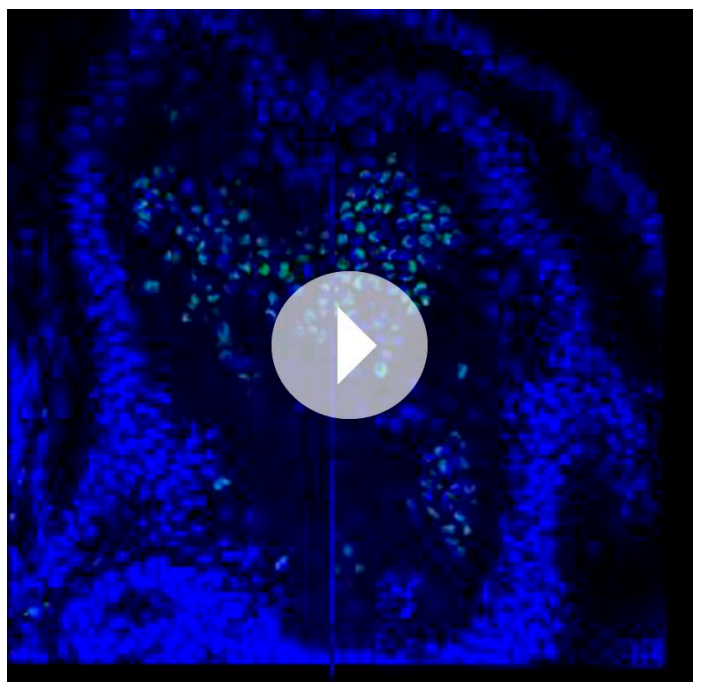

Video 1. Showing multilayered arrangement of myoblasts (related to Figure 3). 3D reconstruction of third instar wing disc showing AMP lineage stained with anti-Twi (green) and all disc nuclei stained using TOPRO (blue) showing stacked arrangement. DOI: 10.7554/eLife.03126.006
Taken together, these findings indicate that proliferation in the AMP lineages occurs in two phases during larval development. In early larval development (first and second instar), AMPs undergo a marked amplification in cell number through symmetric divisions. These divisions are oriented parallel to the wing disc epithelium and hence the AMPs form a monolayer of proliferating cells that remain in contact with the disc epithelium. In late larval development (third instar) AMPs switch to an asymmetric mode of proliferative cell division in which they self-renew and generate postmitotic daughter cells. These asymmetric divisions are oriented obliquely to the wing disc surface such that the self-renewing daughter cells remain in contact with the disc epithelium while the postmitotic daughters become located in the more distal layer of non-replicating myoblasts.

\section{Notch signaling is required for proliferative activity of AMP lineages}

Previous work has shown a requirement for Notch (N) in adult flight muscle development; in $\mathrm{N}$ mutants a depletion of wing disc myoblasts is seen (Anant et al., 1998). This suggests that $\mathrm{N}$ signaling might be involved in the proliferative activity of AMP lineages. To investigate this further, we first determined if $\mathrm{N}$ is expressed in the (Twi-positive) cells on the larval wing disc using an anti-NICD (Notch Intracellular Domain) antibody. Immunocytochemical analysis shows that all of the Twi-positive cells are co-immunolabeled by the anti-NICD antibody (Figure 5A-C, $\mathbf{F}-\mathrm{H}$ ). This co-immunolabeling was seen in all larval instar stages and in all myogenic cell layers associated with the wing disc.

To determine the role of $\mathrm{N}$ in these Twi-positive cells, we used the Dmef2-Gal4 driver together with UAS-N RNAi to down-regulate $\mathrm{N}$ in the AMPs and then assayed mitotic activity using $\mathrm{PH} 3 \mathrm{immu}$ noreactivity in late third instar stage. (Gal80 ts was used to limit N-RNAi to the second and third larval instar to avoid lethality.) A significant reduction in the number of mitotically active cells was observed; in the third instar stage only half the number of $\mathrm{PH}$-positive cells were seen in knockdown vs control experiments (Figure 5D). Similar findings were obtained in experiments in which a dominant negative form of $\mathrm{N}$ was expressed using the Dmef2-Gal4 driver (data not shown). Both the number and the layered organization of the ensemble of Twi-positive cells on the wing disc were reduced in these knockdown experiments (Figure 5I-K). Conversely, overexpression of an activated form of $\mathrm{N}$ in the AMP using Dmef2-Gal 4 to drive UAS-NICD in second and third larval instar stages revealed a marked increase in mitotically active cells as assayed in late third instar stage. In these experiments the number 

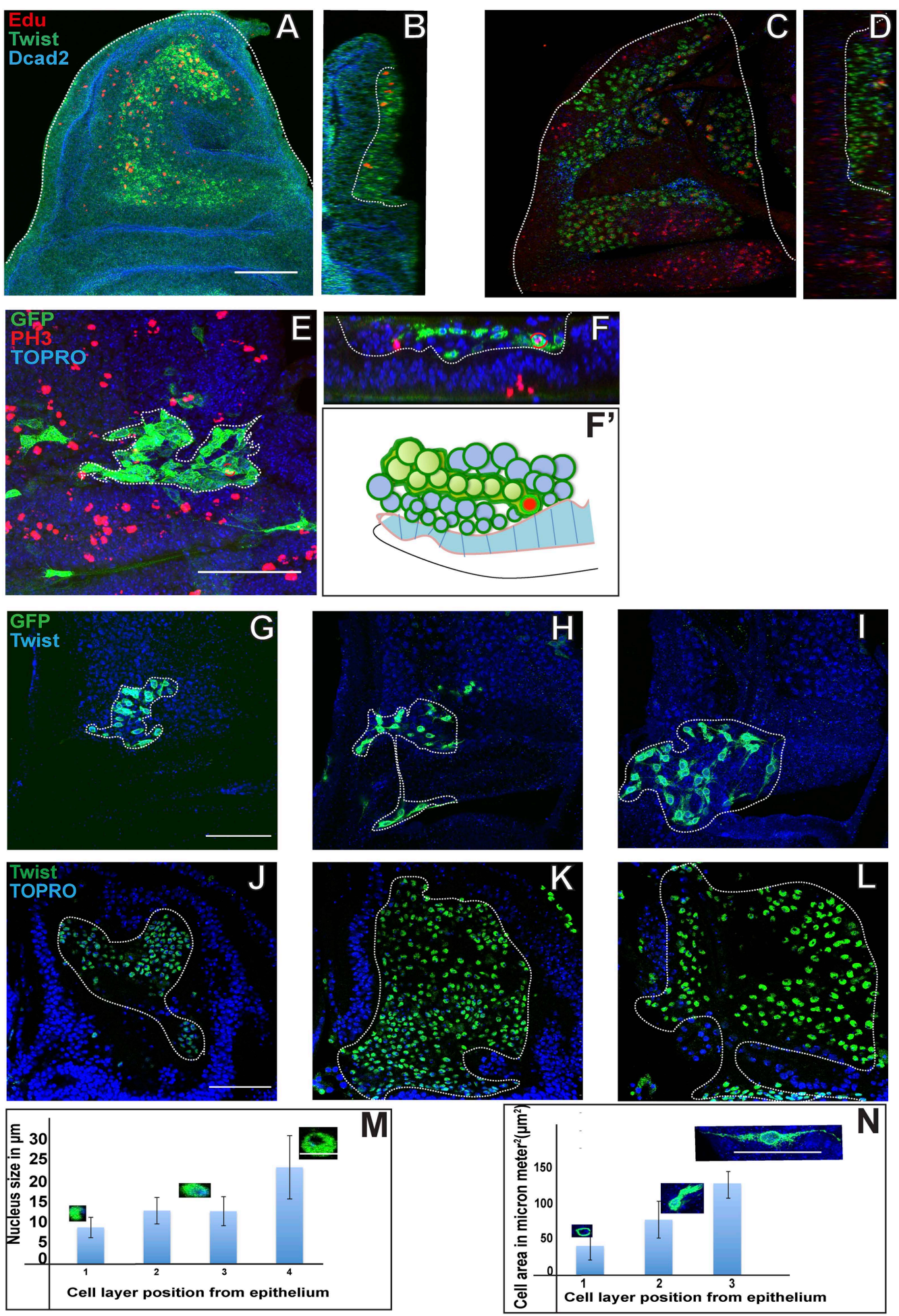

Figure 4. Proliferating AMPs generate clones of post mitotic myoblasts localized in distal layers. (A) Optical section of third instar wing disc dissected after $5 \mathrm{hr}$ Edu, a thymidine analogue (5-ethynyl-2'-deoxyuridine) pulse and stained for Edu (red), Twi (anti-Twist, green) and Dcad2 (anti-Dcad2, blue) revealing presence of Edu in some AMP lineage. $\mathrm{n}=10$. Scale bar, $50 \mu \mathrm{m}$. (B) Sagittal section of presumptive notum region of disc (shown in A) showing Edu labeling of AMP lineage only next to disc epithelium surface. (C) Third instar disc dissected after $5 \mathrm{hr}$ Edu pulse and $48 \mathrm{hr}$ Figure 4. Continued on next page 
Figure 4. Continued

chase in pulse free media and stained for Edu (red), Twi (anti-Twist, green) and Dcad2 (anti-Dcad2, blue) showing labeling in the most distal layer of AMP lineages. $n=12$. (D) Sagittal section of $\mathbf{C}$ showing labeling in maximum labeling in distal layers and minimum in the layer next to epithelium. (E) A MARCM clone generated in second instar stained for GFP (anti-GFP, green) and PH3 (anti-Phosphohistone, red), TO-PRO3 (blue). The clone shows a single $\mathrm{PH} 3$ positive cell (shown in red bracket) in the cluster of other clonal progenies. $n=10$. (F-F') Optical section $(\mathbf{F})$ and schematic $\left(\mathbf{F}^{\prime}\right)$ of figure $\mathrm{E}$, showing the presence of $\mathrm{PH} 3$ positive AMP (red bracket) next to the disc epithelium (dotted line). (G-I) The MARCM clone showing clonal progenies marked with GFP in the proximal most (G), middle $(\mathbf{H})$ and distal most (I) layers with reference to the disc epithelium. The graph $(\mathbf{N})$ shows increase in the cell size from $\mathbf{G}$ to $\mathbf{J} . \mathrm{n}=12$ Scale bar $50 \mu \mathrm{m}$. (K-N) The optical sections of late third instar disc stained for Twi (anti-Twist, green) and TO-PRO3 (blue) and the quantitation (M) showing trend of increase nuclear size in the layers distal most in reference to epithelium. $n=10$. Scale bar $50 \mu \mathrm{m}$.

DOI: 10.7554/eLife.03126.007

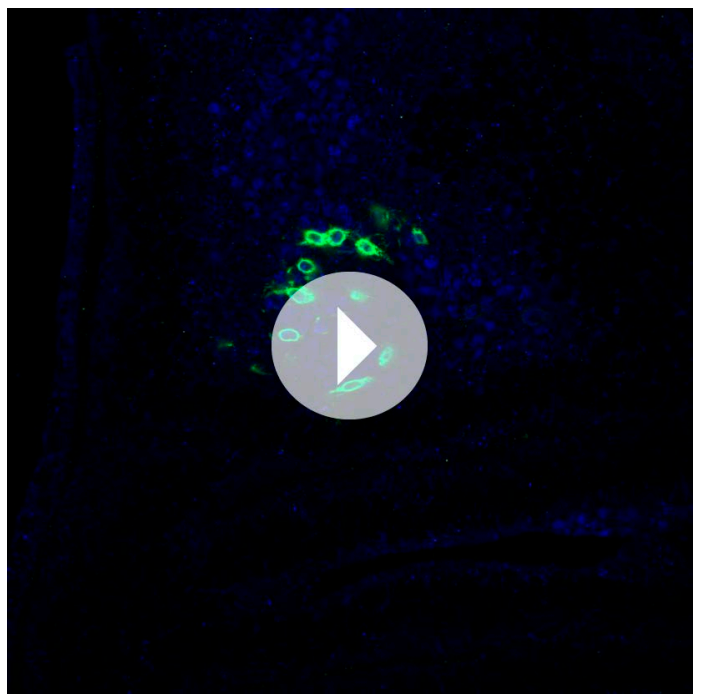

Video 2. MARCM clone showing variation of cell size (related to Figure 4). 3D reconstruction of AMP lineage marked by membrane tethered GFP (mCD8::GFP) revealing size differences in the myoblasts at different distances from disc epithelium. All nuclei marked by TOPRO (blue)

DOI: 10.7554/eLife.03126.008 of PH3-positive cells in the overexpression experiments was approximately twice as large as in controls (Figure 5E). Correspondingly, both the number and the layered organization of the Twi-positive cells on the disc were increased in these overexpression experiments (Figure $5 \mathbf{L}-\mathbf{N}$ ). Interestingly, in contrast to wild type controls, mitotically active cells were occasionally seen in more distal cell layers in $\mathrm{N}$ overexpression experiments (data not shown).

These findings indicate that proliferative activity in the AMP lineages during larval development is dependent on $\mathrm{N}$ signaling. Since mitotic activity in the wild type is only seen in the layer of cells adjacent to the wing disc this further implies that $\mathrm{N}$ signaling acts to effect proliferation in the AMPs in this layer.

\section{Serrate located in the wing disc epithelium is necessary for AMP proliferation}

The $\mathrm{N}$ ligand Serrate (Ser) has been shown to be present in the dorsal (presumptive notum) region of the wing disc in which the AMP lineages develop (Speicher et alo, 1994). This suggests that Ser might be the ligand for the $\mathrm{N}$ signaling required in AMP proliferation. To investigate this, we first determined if Ser expression in the disc epithelium occurs in close proximity to AMPs in the adjacent myogenic cell layer. Immunocytochemical labeling of the wing disc epithelium (with anti-DCad2) and of the AMP lineages (with anti-Twi) together with a Ser-specific marker (Ser-LacZ) reveals a close apposition of Ser-expressing wing disc cells and the AMP cell layer immediately adjacent to the disc epithelium (Figure 6A-H'; Video 4). This close apposition was observed in all larval stages.

The proximity of Ser in the wing disc epithelium to mitotically active AMPs suggests that epithelial Ser could be the ligand that activates $N$ signaling in the AMPs. To investigate if wing disc-derived Ser is required for $\mathrm{N}$-mediated proliferative activity of AMPs, we conditionally knocked down Ser specifically in the dorsal wing disc region of second instar stages with Ap-Gal4 driving UAS-Ser RNAi using the TARGET system (McGuire et al., 2003; Martín and Morata, 2006) and assayed mitotic activity based on PH3 immunolabeling in the late third instar stage (The Ap-Gal4 Gal4 expression in other regions in the larva has no direct effect on these results when perturbation were performed during mentioned time scale). A marked reduction in the number of mitotically active cells was observed; in the third instar stage less than half the number of PH3-positive cells were seen in knockdown vs control experiments (Figure 6l). 


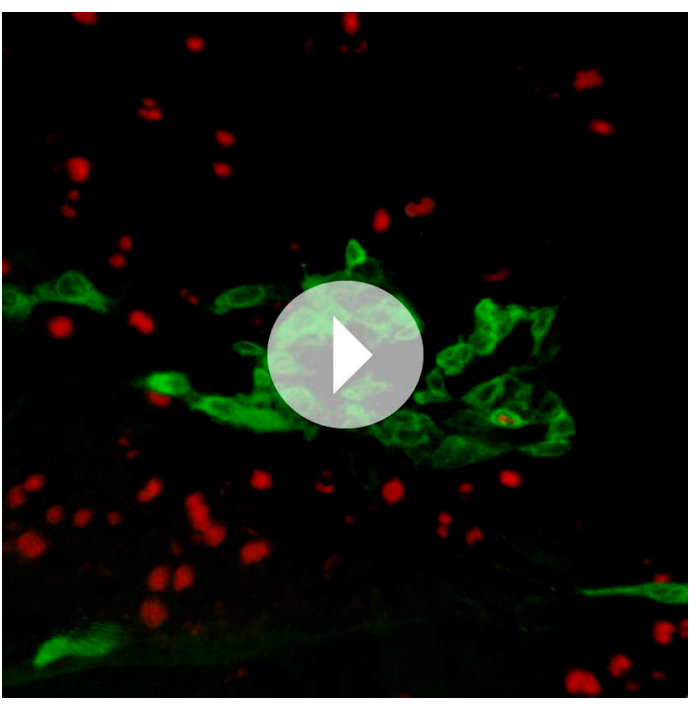

Video 3. MARCM clone showing PH3 association with respect to layers (related to Figure 4). Single cell in a clone (anti-GFP, green) showing active mitotic division (anti-Phospho histone 3, red). DOI: 10.7554/eLife.03126.009
These results indicate that the Ser located in the wing disc epithelium is required for the mitotic activity of cells in the adjacent AMP layer. Given the concomitant requirement of $\mathrm{N}$ for mitotic activity of these cells, this implies that epithelial Ser serves as a ligand for the activation of $\mathrm{N}$ receptor-mediated signaling in the myogenic AMP lineages. This in turn suggests that the Serexpressing cells in the wing disc epithelium represents a transient signaling niche for $\mathrm{N}$ activation of AMP proliferation.

\section{Numb expressed in the third instar stage is required for asymmetric cell divisions in AMP lineages}

While the activation of $\mathrm{N}$ signaling by $\mathrm{Ser}$ is required for mitotic activity in AMP lineages, it is unlikely to mediate the switch from symmetric to asymmetric divisions that occurs during late larval development, since expression of $\mathrm{N}$ and Ser in these lineages occurs throughout larval development. Numb (Nb) is an intracellular protein, which binds to $\mathrm{N}$ and causes degradation of this receptor ultimately leading to down-regulation of $\mathrm{N}$ signaling (Frise et al., 1996; Couturier et al., 2012). Previous work on mitotic activity of muscle progenitors during embryonic development has shown that $\mathrm{Nb}$ acts in asymmetric cell division by down regulating $\mathrm{N}$ activity in one of the progenitor's two daughter cells; in this cell downregulation of $\mathrm{N}$ activity results in specification of muscle cell fate, while continued $\mathrm{N}$ activity in the other daughter cell maintains progenitor cell fate (Ruiz-Gómez et al., 1997; Carmena et al., 1998).

To determine if $\mathrm{Nb}$ is involved in asymmetric cell divisions of AMPs we first carried out immunolabeling studies of $\mathrm{Nb}$ expression during different larval instar stages. These experiments show that $\mathrm{Nb}$ is expressed in AMP lineages during the third instar stage but not in the two preceding larval stages (data not shown). Throughout the third instar stage, $\mathrm{Nb}$ is expressed in all of the myogenic cells on the wing disc, however expression is strongest in the cells of the more distal layers and relatively weak in the cells adjacent to the disc epithelium; this contrasts with $\mathrm{N}$ expression which is more uniformly distributed in all of these cell layers (Figure 7A-D). The fact that Nb is not expressed in AMP lineages during their symmetric division phase, but is present in these cells during their asymmetric division phase suggests that $\mathrm{Nb}$ might be required for the transition to asymmetric cell divisions in AMP lineages.

To investigate this, we carried out twin-spot MARCM labeling experiments and used Dmef2-Gal4 driving UAS-Nb RNAi to knockdown Nb in the proliferating AMP lineages. Clones were induced in the early third instar stage and recovered in the late third instar stage. These twin-spot MARCM clones invariably consisted of two relatively large, differentially (RFP and GFP) labeled daughter cell clones that were similar in size and cell number indicative of symmetric divisions (Figure $7 F-H$ ). These clones were similar to the corresponding twin-spot clones obtained in wild type first and second instar stages but strikingly different from those obtained in the wild type third instar (see Figure 2). This indicates that in the absence of $\mathrm{Nb}$, cell divisions in AMP lineages are symmetric and not asymmetric in the third instar stage, implying that $\mathrm{Nb}$ expression is necessary for the transition from the symmetric to the asymmetric division mode manifest in the third instar.

Given that $\mathrm{Nb}$ inhibits $\mathrm{N}$ signaling, upregulation of $\mathrm{N}$ should have similar phenotypic effects as downregulation of $\mathrm{Nb}$. To investigate this, we repeated the twin-spot MARCM labeling experiments but used Dmef2-Gal4 driving UAS-NICD to constitutively activate $\mathrm{N}$ in the proliferating AMP lineages. Clones induced in the early third instar stage and recovered in the late third instar stage again consisted of two relatively large, differentially labeled daughter cell clones that were similar in size and cell number indicating a symmetric division mode (Figure $7 \mathrm{II}-\mathrm{K}$ ). Since both downregulation of $\mathrm{Nb}$ and constitutive upregulation of $\mathrm{N}$ lead to continued symmetric cell divisions in the third instar stage, we postulate that $\mathrm{Nb}$, through downregulation of $\mathrm{N}$, is required for asymmetric proliferative divisions in the AMP lineages. 

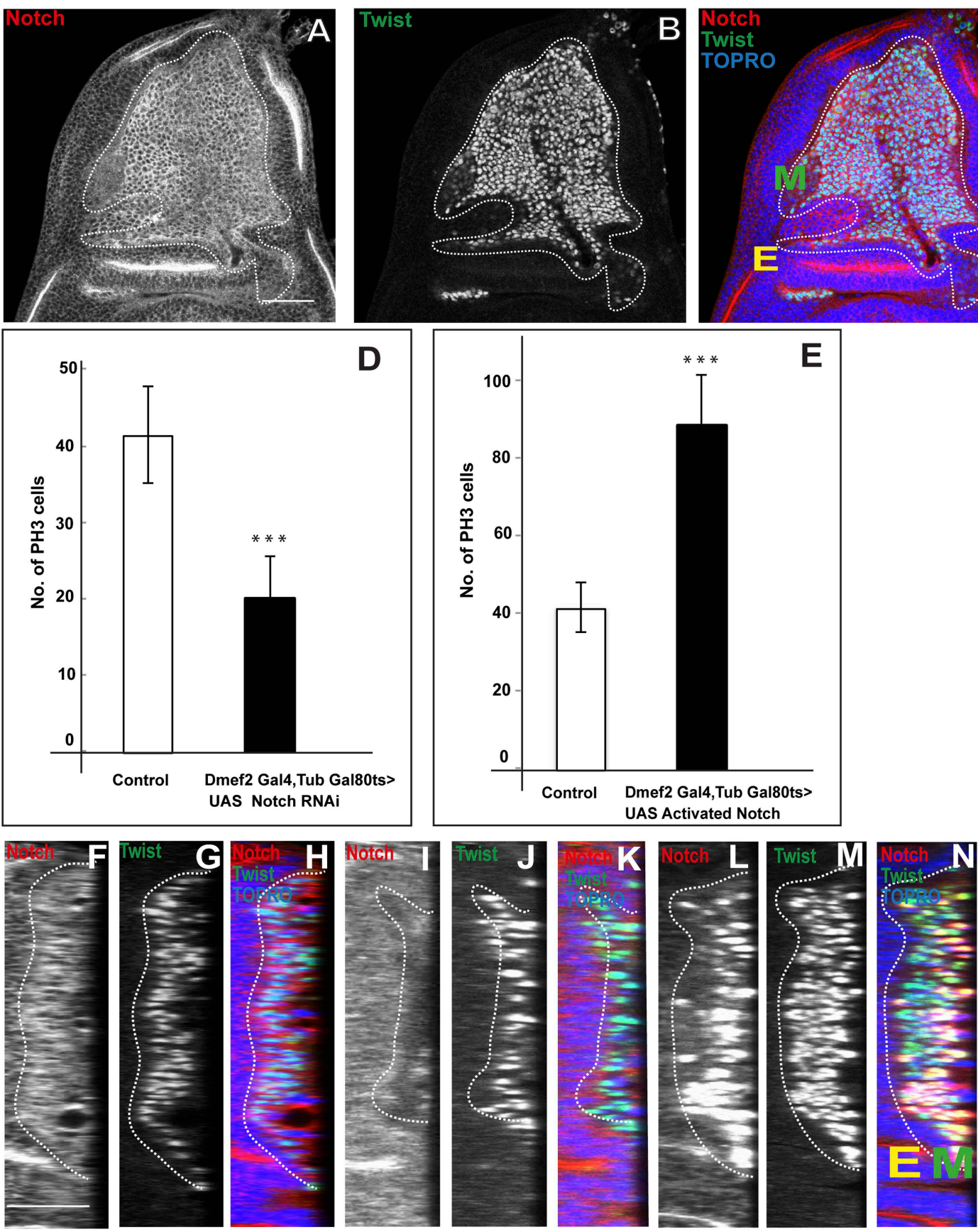

Figure 5. Notch signaling is required for proliferative activity of AMP lineages. (A-C) Late third instar disc stained for Notch (anti-Notch intracellular C-terminal domain, [NICD, red]), Twi (anti-Twist, green) and TO-PRO3 (blue). In this figure, E denotes disc-epithelium and M is for mesoderm (green). (D-E) Quantification of number of PH3 positive AMPs in the Notch donwregulation using Dmef2-Gal4, TubGal80ts > UAS Notch RNAi (D) and Notch up regulation using Dmef2-Gal4, TubGal80ts > UAS NICD background (E). For both experiments Gal80 repression was relieved from early second instar till late third instar by shifting from $18^{\circ} \mathrm{C}$ to $29^{\circ} \mathrm{C}$. All graphs are Mean \pm Standard Error (Student's $t$ test). $n=12$. p-values $<0.001$. (F-N) Optical section of wing discs stained for Notch (anti-Notch intracellular C-terminal domain, (NICD, red), Twi (anti-Twist, green) and TO-PRO3 (blue) in control (F-H) (Dmef2-Gal4, TubGal80ts > Canton-S), in Dmef2-Gal4, TubGal80ts > UAS Notch RNAi (I-K), Dmef2-Gal4, TubGal80ts > UAS NICD (L-N). The multistratified layered arrangement of AMP lineages is lost in Notch down regulation $(\mathbf{J})$ while in Notch up regulation $(\mathbf{M})$ it increases with respect to number of layers, in comparison to control (G). Gal80 repression was relieved from early second instar till late third instar by shifting from $18^{\circ} \mathrm{C}$ to $29^{\circ} \mathrm{C}$. $n=20$. Scale bar, $50 \mu \mathrm{m}$. E denotes disc-epithelium and $\mathrm{M}$ is for mesoderm (marked by anti-Twist, green).

DOI: 10.7554/eLife.03126.010 

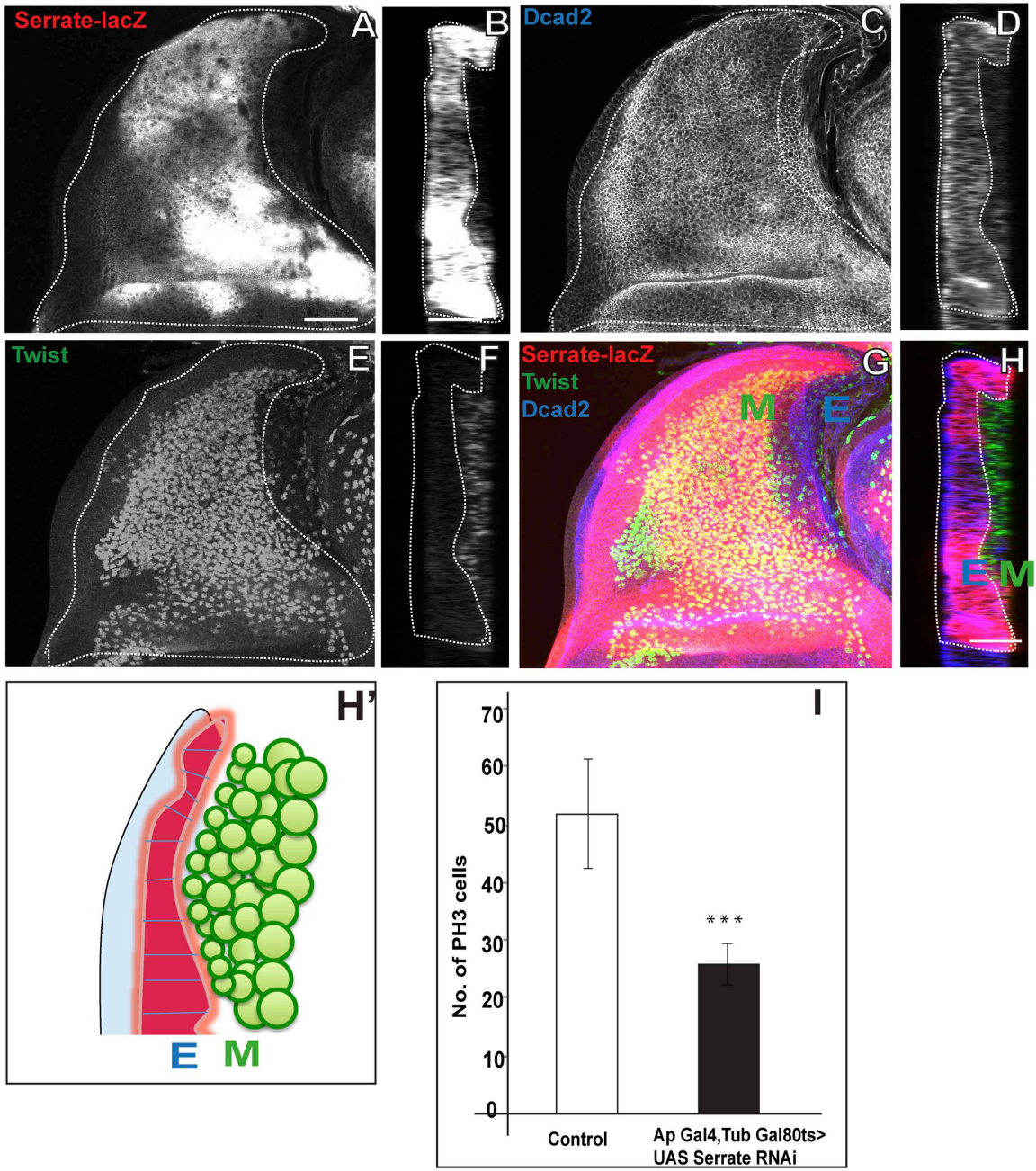

Figure 6. Serrate located in the wing disc epithelium is necessary for AMP proliferation. (A) Serrate-lacZ (anti-beta Gal), a reporter for Serrate expression, visualized in disc epithelium of the late third instar. (B) Saggital section shows Serrate being expressed specifically in the disc epithelium. (C-D) Dcad2 expression (anti-Dcad2) marking the disc epithelium. (E-F) AMP lineages viewed by using Twi staining (anti-Twist). (G-H) The merge shows the expression of Serrate (red) exclusively in the disc epithelium as Dcad2 (blue) and in close proximity of first layer AMP lineages. E-wing disc epithelium, M-AMP lineages. Scale bar $50 \mu \mathrm{m}$. $\mathrm{H}^{\prime}$-Schematic depiction of expression patterns of Serrate, Dcad2 and Twist showing only one layer of AMP out of 3-4 (As shown in F) is in direct contact with Serrate producing epithelium surface. (I) Quantification of number of PH3 positive AMPs in disc epithelium specific Serrate knock down using Ap Gal4 driving UAS Serrate RNAi. Gal80 repression was relieved from early second instar till late third instar by shifting from $18^{\circ} \mathrm{C}$ to $29^{\circ} \mathrm{C}$. All graphs are Mean \pm Standard Error (Student's $t$ test). $\mathrm{n}=15$. p-values $<0.001$

DOI: 10.7554/eLife.03126.011

\section{Wingless signaling from the wing disc epithelium induces Numb expression in third instar AMP lineages}

While above mentioned experiments indicate that $\mathrm{Nb}$ expression in AMP lineages is important for the transition from symmetric to asymmetric proliferation divisions, they do not identify the signal that controls the onset and maintenance of $\mathrm{Nb}$ expression in the third instar stage. Wingless $(\mathrm{Wg})$ is a signaling protein that can act to control developmental patterning and growth (Sharma and Chopra, 1976; Zecca et al., 1996; van Amerogen and Nusse, 2009). During larval development of the wing disc, Wg is expressed in the prospective wing blade region in all instar stages, however, expression in the prospective notum region, in which the developing AMP lineages reside, is only manifest from the 


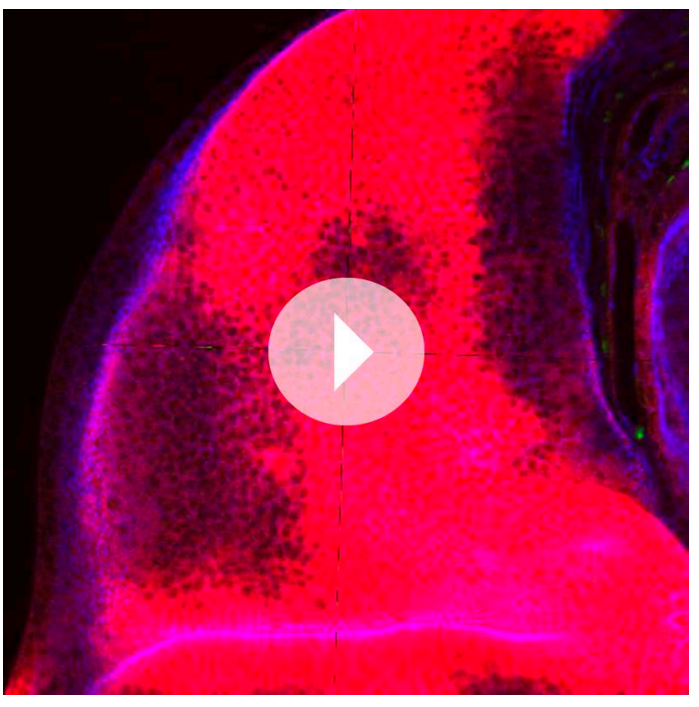

Video 4. Serrate staining in epidermis and close association with myoblasts (related to Figure 6). Serrate- lacZ (anti-beta-gal, red) showing serrate expression in wing disc epithelium (anti-Dcad 2, blue) and only one layer of myoblasts are in contact with epithelium due to multilayered arrangement. DOI: 10.7554/eLife.03126.012

implying that inductive $\mathrm{Wg}$ signaling from the notum region of the wing disc affects all myogenic cells in the third larval instar (Figure 8I-N). We next carried out similar Wg loss-of-function experiments together with $\mathrm{Nb}$ immunolabeling to investigate if inductive $\mathrm{Wg}$ signaling is required for onset and maintenance of $\mathrm{Nb}$ expression in the third instar myogenic cells. Wg loss-of-function in the wing disc notum resulted in complete loss of $\mathrm{Nb}$ expression in all Twi-positive cells (Figure 80-V). We conclude that $\mathrm{Wg}$ signaling in the wing disc notum is required for induction and maintenance of $\mathrm{Nb}$ expression in the myogenic AMP lineages in the third larval instar stage.

In addition to its effects on $\mathrm{Nb}$ expression, notal $\mathrm{Wg}$ loss-of-function also results in the reduction of mitotic activity in the AMP lineages. Thus, both the number of $\mathrm{PH} 3$ positive cells and the extent of the multilayered organization are reduced in Wg downregulation compared to wild type (Figure 9A-J, Figure 9-figure supplement 1). To understand the possible direct link of the induction of Wg signaling and change in the orientation of the cell division, we down regulated Wg using DN-TCF with Dmef2-Gal4 and probed for division axis using CNN-GFP. The axis of the division in this background is parallel to the epithelium, similar to that seen in the early instars (Figure 9-figure supplement 2). Wg, in the presumptive notum is expressed only from the late second instar (Alexandre et al., 2014), therefore this result shows that down-regulation of $\mathrm{Wg}$ prevents the switch in cell-division orientation from parallel to the epithelium to perpendicular to the epithelium. This suggests that Wg signaling from the wing disc niche might have dual effects on neighboring myogenic cells, namely the induction of $\mathrm{Nb}$ expression and the regulation of proliferation.

\section{Discussion}

Early studies in Calliphora (Crossley, 1972) and elegant clonal analysis and transplantation experiments in Drosophila (Brower et al., 1981; Lawrencea, 1982) established mesodermal cells attached to the wing imaginal disc as the source of muscle progenitors for the large indirect flight muscles of the adult thorax of dipteran insects. These 'adepithelial' cells were subsequently shown to express the mesodermal marker Twist (Thisse et al., 1988; Currie and Bate, 1991; Fernandes et al., 1991). Twist labeling of the AMPs on the late-third instar wing imaginal disc reveal a large number of cells (Currie and Bate, 1991; Fernandes et alo, 1991), which this study identifies as about 2500. The haltere (T3) disc and the wing (T2) disc, start off with a similar numbers of myoblasts in the late embryo. Studies on muscle development in the 'four-winged fly', where the function of the homeotic gene Ultrabithorax 

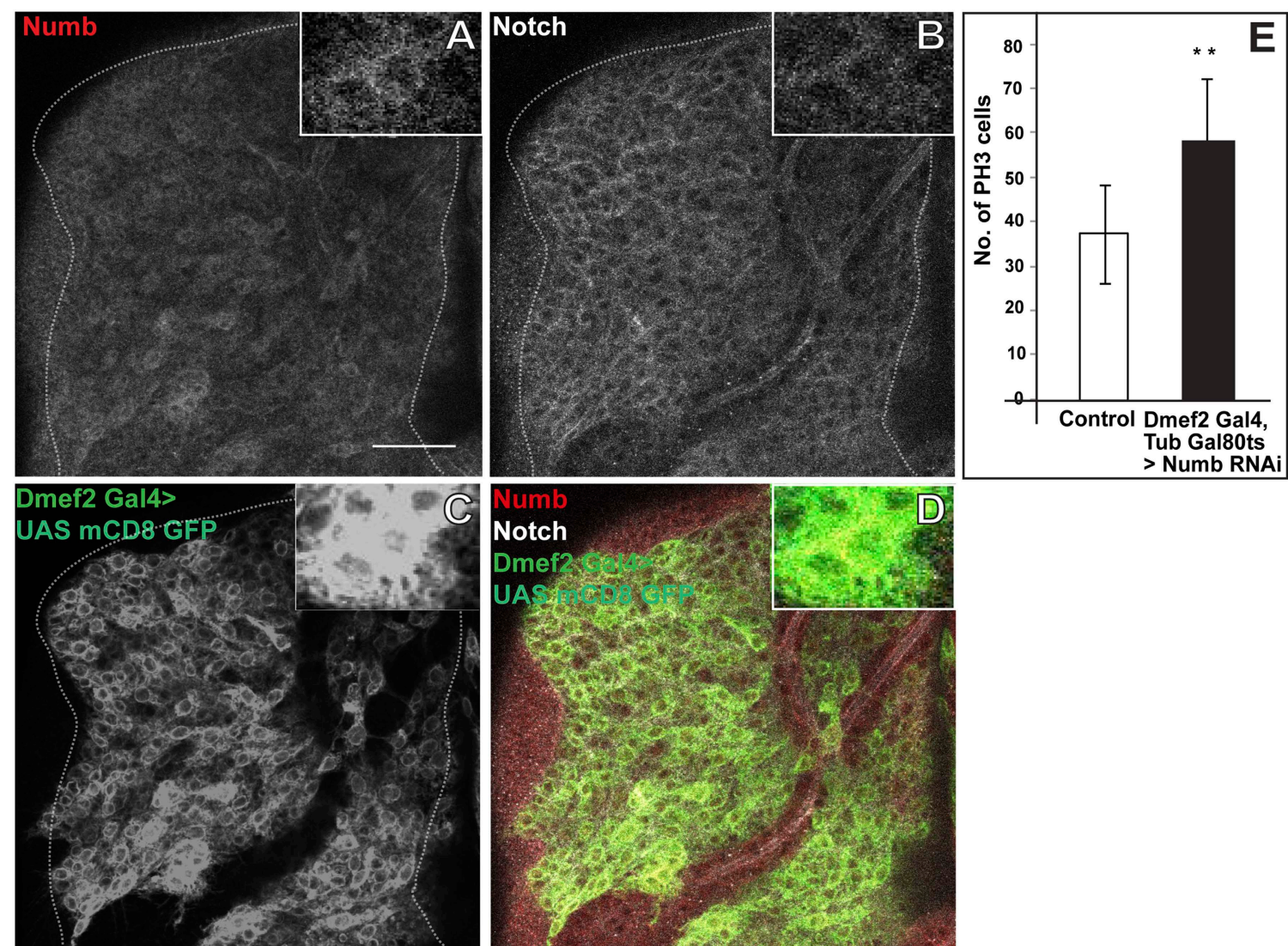

$>$ Numb RNA
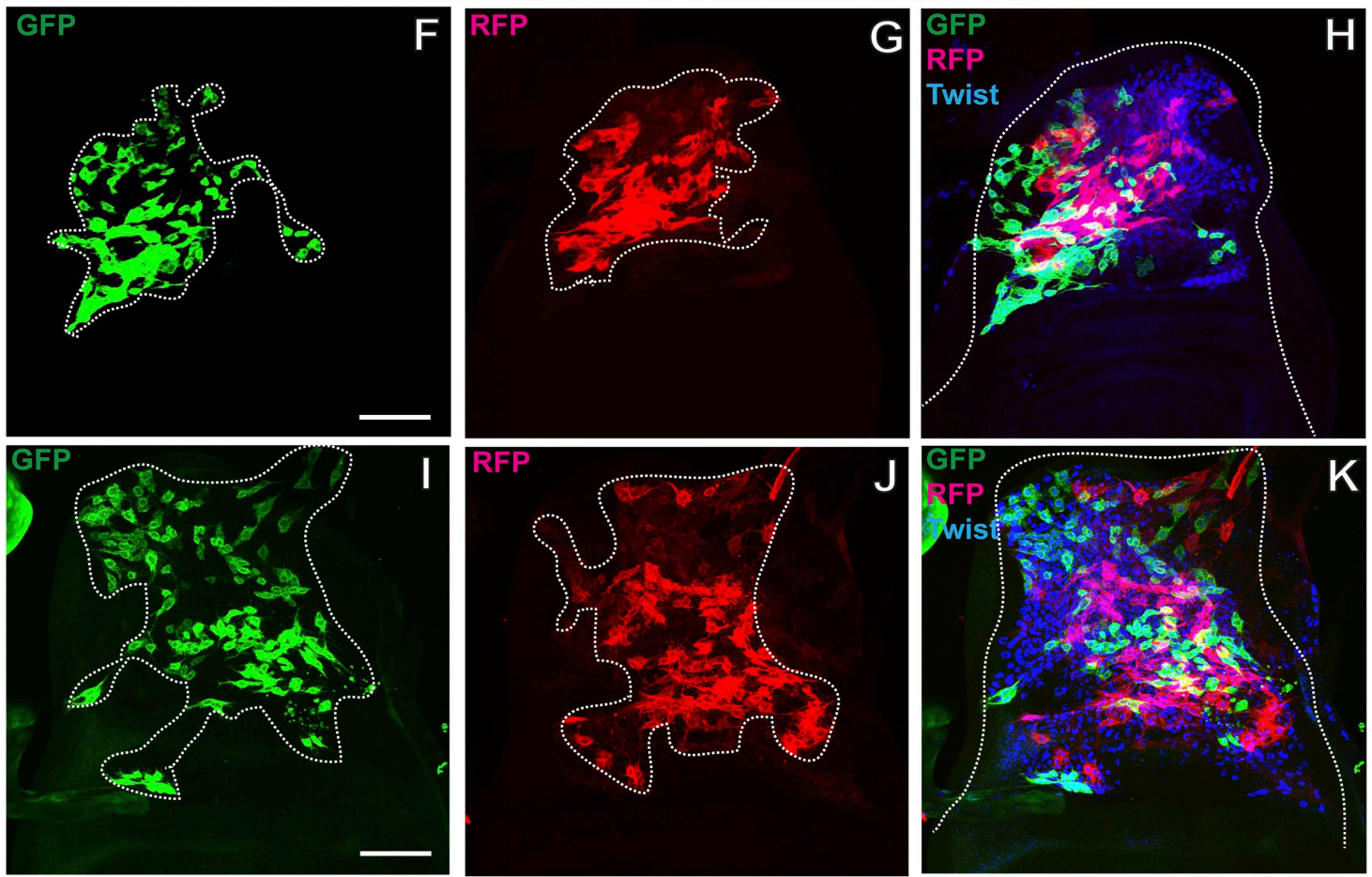

Figure 7. Numb expressed in the third instar stage is required for asymmetric cell divisions in AMP lineages. (A-D) The late third instar disc stained for expression of Numb (anti-Numb, red), Notch (anti-NICD, white) and Dmef2-Gal4 > UAS mCD8GFP (anti-GFP, green). N = 6.The expression of Numb can be seen in patches $(\mathbf{A})$ in contrast to Notch (B) which stains most of the AMP lineages marked by using Dmef2-Gal4 > UAS mCD8GFP (C). The merge (D) Figure 7. Continued on next page 
Figure 7. Continued

shows the expression of Notch (white) in myoblasts lineage (green) along with Numb (red). (E) Quantification of number of PH3 positive AMPs in Numb knock down (Dmef2-Gal4, TubGal80ts > UAS Numb RNAi) showing significant increase in the total proliferating AMPs. Gal80 repression was relieved from early third instar till late third instar by shifting from $18^{\circ} \mathrm{C}$ to $29^{\circ} \mathrm{C}$. All graphs are Mean \pm Standard Error (Student's $t$ test). $n=10$. $p$-values $<0.001$. (F-K) Twinspot MARCM in Numb RNAi (F-H) and in Notch upregulation (I-K) backgrounds, generated in early third instar, showing loss of asymmetry or symmetric clones in contrast to wild type asymmetric clone as shown in Figure 2D-F. $n=5$. Scale bar, $50 \mu m$.

DOI: 10.7554/eLife.03126.013

(Ubx) is removed from the haltere disc, showed that AMP population is controlled by the disc-epidermis. When the haltere ectoderm is transformed to T2 with the AMPs continue to retain a T3 identity, the AMPs nevertheless transform to a T2 population size (Fernandes et al., 1994; Roy et al., 1997). Despite these early studies examining the role of signaling from the disc-ectoderm (Sudarsan et al., 2001) in flight muscle specification (Schnorrer et al., 2010; Schönbauer et al., 2011), the mechanism of population expansion have not been hitherto investigated. This is an important general problem as it addresses how diversification of internal tissues in segmented animals takes place.

Our results show that, in early development the AMPs on the wing-disc go through a phase of exponential amplification from about 10 cells to 250 . This amplification, where the daughters of each stem are both stem cells, takes place by the division of cells parallel to the disc-epithelium stem-cell niche and requires Ser- $\mathrm{N}$ signaling. As the expression of $\mathrm{Wg}$ in the presumptive notum known to occur in the late second instar larva (Tomoyasu et al., 2000; Alexandre et al., 2014) changes in the AMPs proliferative behaviour are seen. A switch to asymmetric cell- division results in a 'self-renewed' stem cell and a post-mitotic sibling poised to differentiate to contribute to multinucleate muscles. The plane of cell division is no longer parallel to the ectoderm, resulting a stratified layer of cells, with the discproximal layer consisting of stem cells.

Our findings support a model in which the embryonically generated AMPs acting as postembryonic muscle stem cells generate adult-specific myoblasts in a two-step process (Figure 10). AMPs initially manifest a symmetric cell division mode, which serves to amplify the progenitor pool in the first and second larval stages, and subsequently transit to an asymmetric cell division mode during the third larval stage during which they self-renew and generate the postmitotic myoblasts required for adult muscle formation. In both steps, signaling is required from the notum region of the wing disc epithelium, which acts as a transient niche. Thus, Ser localized in epithelial cells of the disc is necessary for activation of $\mathrm{N}$ signaling in AMPs throughout larval development, and diffusible $\mathrm{Wg}$ from the disc epithelial cells in the third larval instar is necessary for the expression of Nb in AMP lineages and, hence, for their transition from symmetric to asymmetric division modes. The idea of epithelium tissue as transient niche for the regulation of proliferation is something similar to that known in intestinal stem cell (Mathur et al., 2010). In this paper authors provide evidence for the role of progeny of ISC progenitor as a very transient signaling center regulating stem cell proliferation via decapentaplegic.

Since these findings reveal remarkable parallels to other tissue stem cell systems in Drosophila, we consider the disc-associated AMPs to be a novel type of muscle stem cell that orchestrates the early phases of adult flight muscle development in Drosophila. This is a newly identified and key role for $\mathrm{Wg}$ in the presumptive notum. Its other roles include a requirement for expression of the muscle-attachment gene stripe (sr), in the presumptive notum (Ghazi and VijayRaghavan, 2003) and for maintenance of the indirect-flight muscle marker Vg, in AMPs (Sudarsan et alo, 2001).

$\mathrm{Wg}$ causes this switch in cell- division pattern by the transcription of $\mathrm{Nb}$ in the stem-cell layer, and its asymmetric distribution by well-established mechanisms (Skeath and Doe, 1998; Couturier et alo, 2012) thereby inhibiting Notch activation in the post mitotic progeny.

Many types of tissue stem cells, in a manner similar to that shown here for the AMPs, have the capability of proliferating through symmetric cell divisions, thereby expanding the stem cell pool, and then switching to asymmetric cell divisions. In Drosophila optic lobe development, a set of 30-40 epithelial progenitor cells generated in the embryo begin to proliferate in a symmetric cell division mode during early larval development to generate a neuroepithelial cell pool of approximately 700 cells. These neuroepithelial cells sequentially transform into neuroblasts, which generate differentiated neural cells of the medulla through asymmetric divisions. This transition from symmetric to asymmetric divisions involves cellular events such as the reorientation of the mitotic spindle and multiple molecular signaling processes including the dynamic regulation of Notch signaling (Egger et al., 2010, 2011). Since this transition from symmetrical 'proliferative' divisions to asymmetrical 'differentiative' divisions 

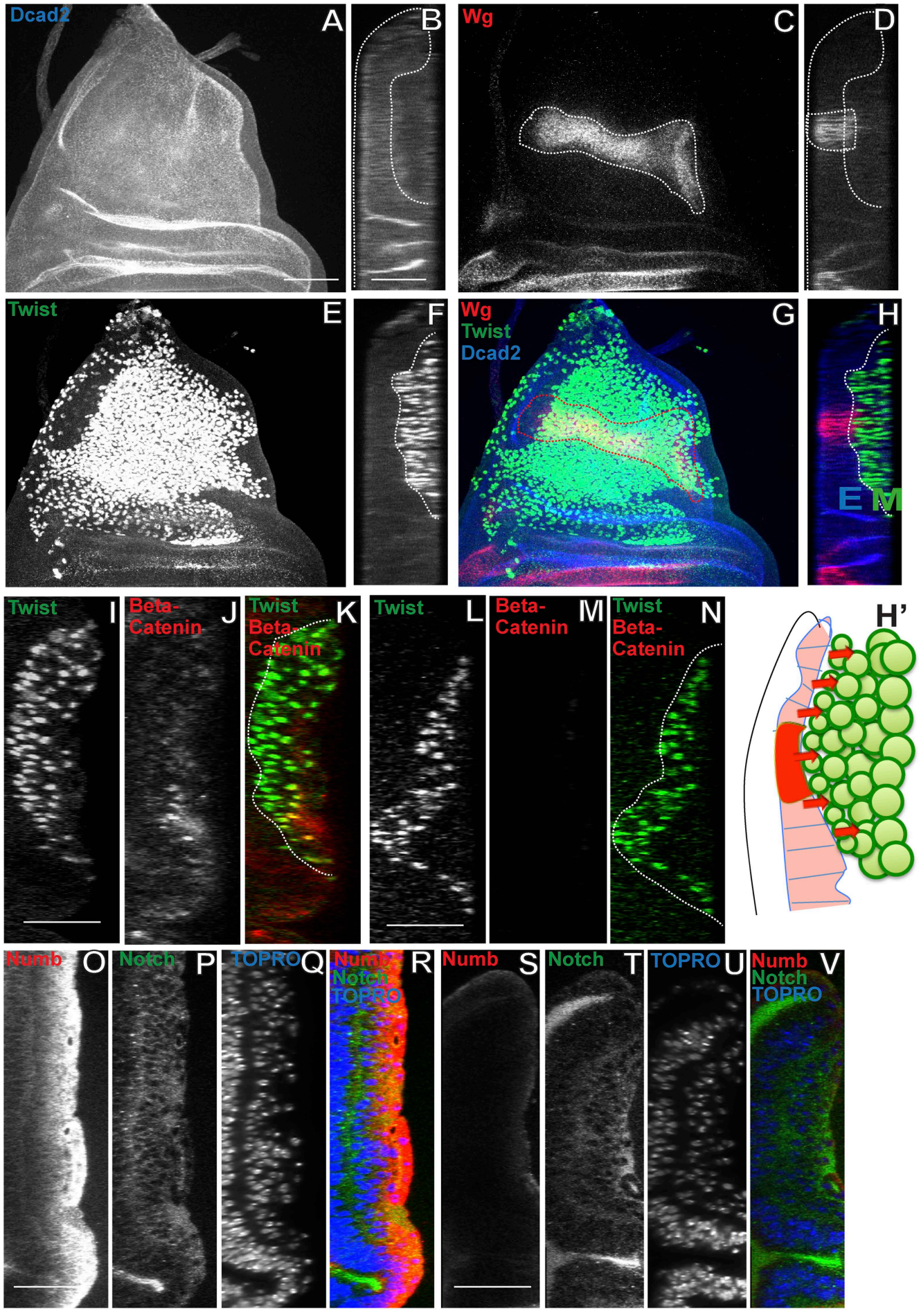

Figure 8. Wingless signaling from the wing disc epithelium induces Numb expression in third instar AMP lineages. (A-H) Third instar wing disc stained for Dcad2 (anti-Dcad2, Blue), Wg (anti-Wg, red) and Twi (anti-Twist, green) demonstrating a prominent longitudinal stripe of $\mathrm{Wg}$ expression in disc epithelium. $n=20$. $\left(\mathbf{H}^{\prime}\right)$ Schematic of the merge $(\mathbf{H})$ depicting disc epithelium, as a source of $\mathrm{Wg}$ production and dispersal subsequently leading to $\mathrm{Wg}$ signaling (red arrows) activated in all AMPs. (I-N) AMP lineages (anti-Twi, green) stained for Beta-catenin (down stream molecule of Wg pathway) (anti-Beta catenin, red) in Canton-S (I-K) and in Wg(ts)/Wg(Sp-1) alleles (loss of Figure 8. Continued on next page 
Figure 8. Continued

function alleles of $\mathbf{W g}$ gene) (L-N). Loss of Beta-catenin in $\mathrm{Wg}(\mathrm{ts}) / \mathrm{Wg}(\mathrm{Sp}-1)$ shows absence of $\mathrm{Wg}$ activation in AMPs which also leads to decrease in number of AMP lineages. Presence of Beta-catenin in all AMPs clearly points towards Wg action at a distance far from the disc epithelium, the source of Wg. $n=10$. (O-R) The optical section of third instar wing disc showing Numb expression (anti-Numb, red) very prominent in the distal layer of AMPs, marked by Notch (anti-Notch, green) (also in Figure 5A-C). (S-V) Wg loss of function (Wg(ts)/Wg(Sp-1)) results in total disappearance of Numb in AMPs. $n=10$. Scale bar, $50 \mu \mathrm{m}$.

DOI: 10.7554/eLife.03126.014

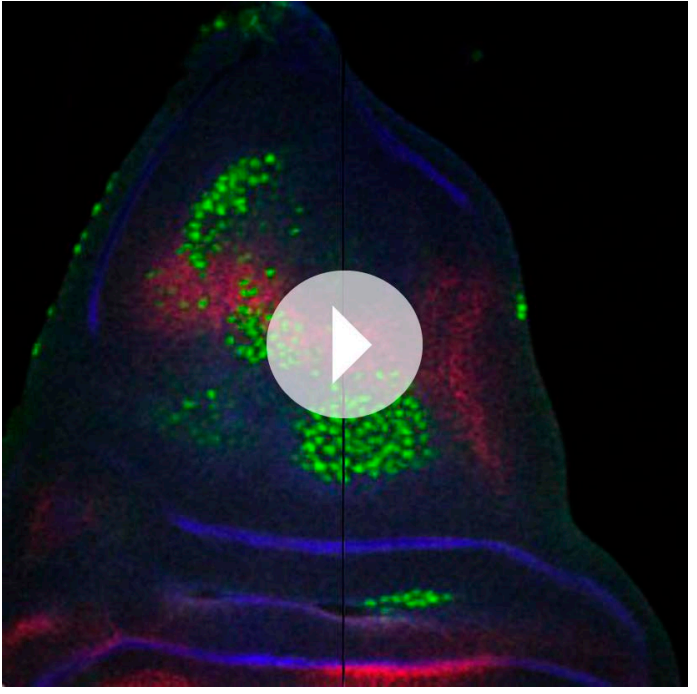

Video 5. Wg staining in Canton-S (related to Figure 8). Late third instar wing disc showing Wg (anti-Wg, red) expression in disc epithelium (anti-Dcad2, blue). The myoblasts forms a close association with Wg expression. DOI: 10.7554/eLife.03126.015

tion of cell-division. This ensures the balance between self-renewal and differentiation as progen which lose contact with the signaling niche, is canalized to a particular fate, whereas those still in contact remain as self-renewing stem cells. In germline stem cell (GSC) in the ovarioles and testis, the contact of stem cells with the tightly regulate stem cell number and coordinated maturation of the progeny to form gametes. Loss of regulation in this axis results in mis-regulated cell number amplification (López-Onieva et al., 2008; Wang et al., 2008; Losick et al., 2011). The intestinal stem cell niche in Drosophila and Mus have a cryptic stem cell niche where a differentiating progeny signal back to the stem cell through EGF signaling, acting as mitogen (Jiang and Edgar, 2009). Neuroblasts in Drosophila are widely considered as neural stem cells as they are shown to go through a series of self-renewing divisions while siblings differentiating to form neurons. Remarkably, this is also seen in the culture, suggesting that this is an intrinsic property and not niche dependent. However, surrounding glia in vivo are proposed to have a regulatory effect on the stem cell abilities of these cells (Chai et al., 2012).

There have also been previous studies in Drosophila that address how stem cell division relates to regulation of population size. It is known from the studies in germline stem cell (Gilboa and Lehmann, 2006) and neural stem cells (Egger et al., 2011) there is change in division rates during larval development. In the early instars there is an amplification that slows down in the third instar. The ovary of adult Drosophila has 16-18 units, ovarioles, which are formed during the larval stages. Each ovariole contains two or three germline stem cells, which are in contact with somatic cells that regulate their establishment, maintenance and differentiation. For example, the developmental origin of the ovaries are the primordial germ cells (PGCs), which till the third instar contribute to most of the cells required for gonad formation and in pupal stage separate into ovarioles (Gilboa and Lehmann, 2006). The numbers of PGCs double every $24 \mathrm{hr}$ after hatching till the mid-third instar after which it slows down, finally forming 100 cells starting from 12 cells (Gilboa and Lehmann, 2006). Such examples and our 

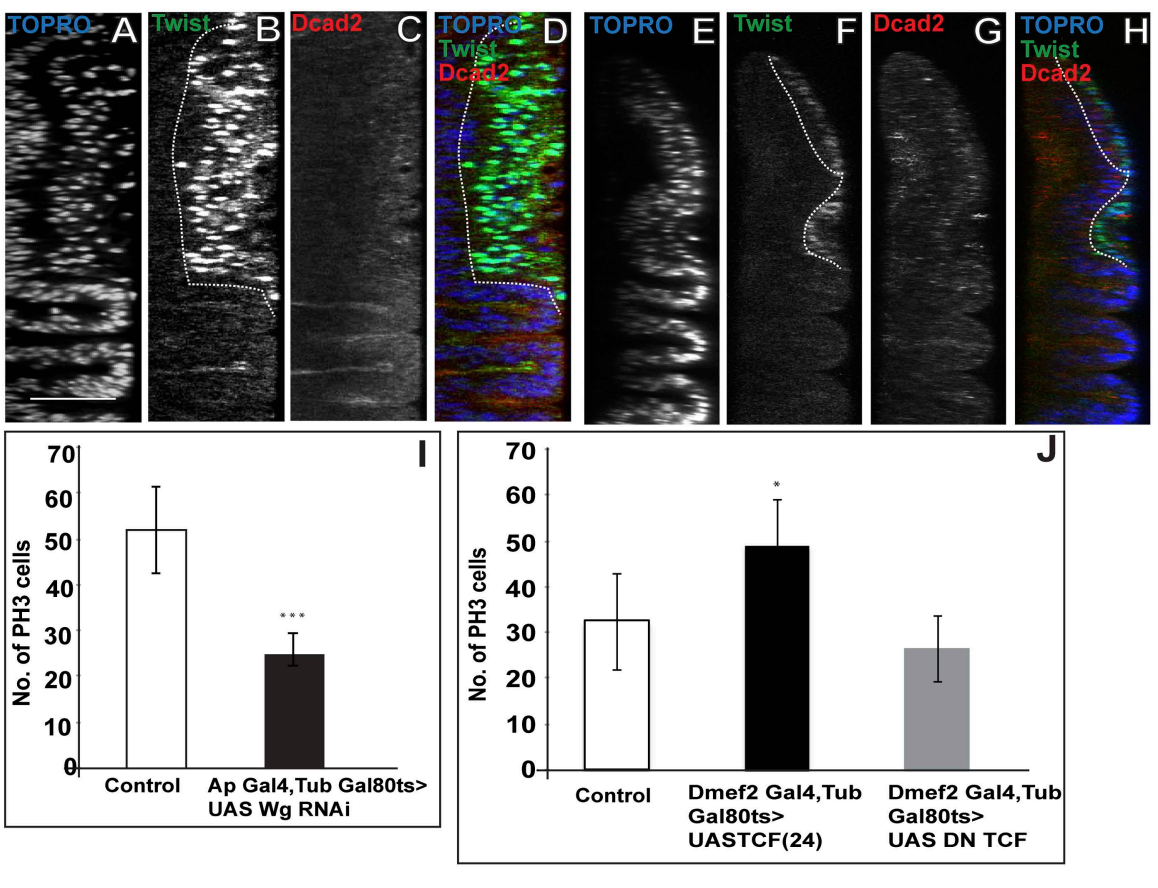
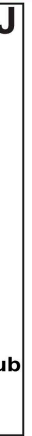

Figure 9. Loss of $\mathrm{Wg}$ results in reduction of mitotic activity and layered arrangement in AMP lineage. (A-H) Third instar wing disc showing loss of multilayered arrangement in Wg loss of function back ground $(\mathrm{Wg}(\mathrm{ts}) / \mathrm{Wg}(\mathrm{Sp}-1))$. In control disc (Canton-S) the multistratified arrangement can be distinctly seen which clearly disappears in Wg loss of function background. $n=8$. (I) Quantification of number of PH3 positive AMPs in epithelium specific Wg knockdown using ApGal4 > UAS Wg RNAi showing significant decreases in comparison to control (Canton-s). All graphs are Mean \pm Standard Error (Student's $t$ test). $p$-value $<0.001, n=10$. (J) AMP specific perturbations (using Dmef2-Gal4) of Wg pathway downstream molecules (TCF) showing changes in mitotic activity in comparison to control. The activation of Wg pathway by overexpressing activated TCF (UAS TCF 24) leads to significant increase in mitotic activity. Gal80 repression was relieved from early second instar till late third instar by shifting from $18^{\circ} \mathrm{C}$ to $29^{\circ} \mathrm{C}$. All graphs are Mean \pm Standard Error (Student's $t$ test). $n=15$.

DOI: 10.7554/eLife.03126.016

The following figure supplements are available for figure 9:

Figure supplement 1. Membrane tethered Wg perturbs multistratified arrangement of AMPs. DOI: 10.7554/eLife.03126.017

Figure supplement 2. Wg down regulation alters division axis of third instar AMPs.

DOI: 10.7554/eLife.03126.018

results tempt us to speculate that an early amplification and a later slowing down maybe a general feature of regulation of stem cell proliferation in Drosophila.

Our results and the underlying mechanisms could also be of general applicability in understanding myogenesis in other contexts. In vertebrates, the Hox identity of the ectoderm could influence proliferation in the mesoderm, in a manner similar to that observed by us, to create the appropriate population of progenitors for the morphogenesis of muscles of very different sizes. Our study suggests the testable hypothesis that vertebrate myoblast pools also develop by symmetric and asymmetric division of muscle progenitor stem cells.

In vertebrate muscle, satellite cells form a quiescent muscle stem-cell population important for the regeneration (Brack and Rando, 2012; Cooper et al., 1999; Le Grand and Rudnicki, 2007; Vasyutina et al., 2007). A recent study (Konstantinides and Averof et al., 2014) of limb regeneration in the crustacean, Parhyale hawaiensis identified satellite like cells (SLCs) expressing Pax3/7 genes expressed in vertebrate muscle satellite cells (Kassar-Duchossoy et al., 2005). This study along with others show a common mesodermal origin of adult muscle stem cells in crustaceans and chordates though the precise developmental origins of these remains a mystery (MAURO, 1961; Bryson-Richardson and Currie, 2008; Sambasivan and Tajbakhsh, 2007). Our study raises the interesting possibility that 


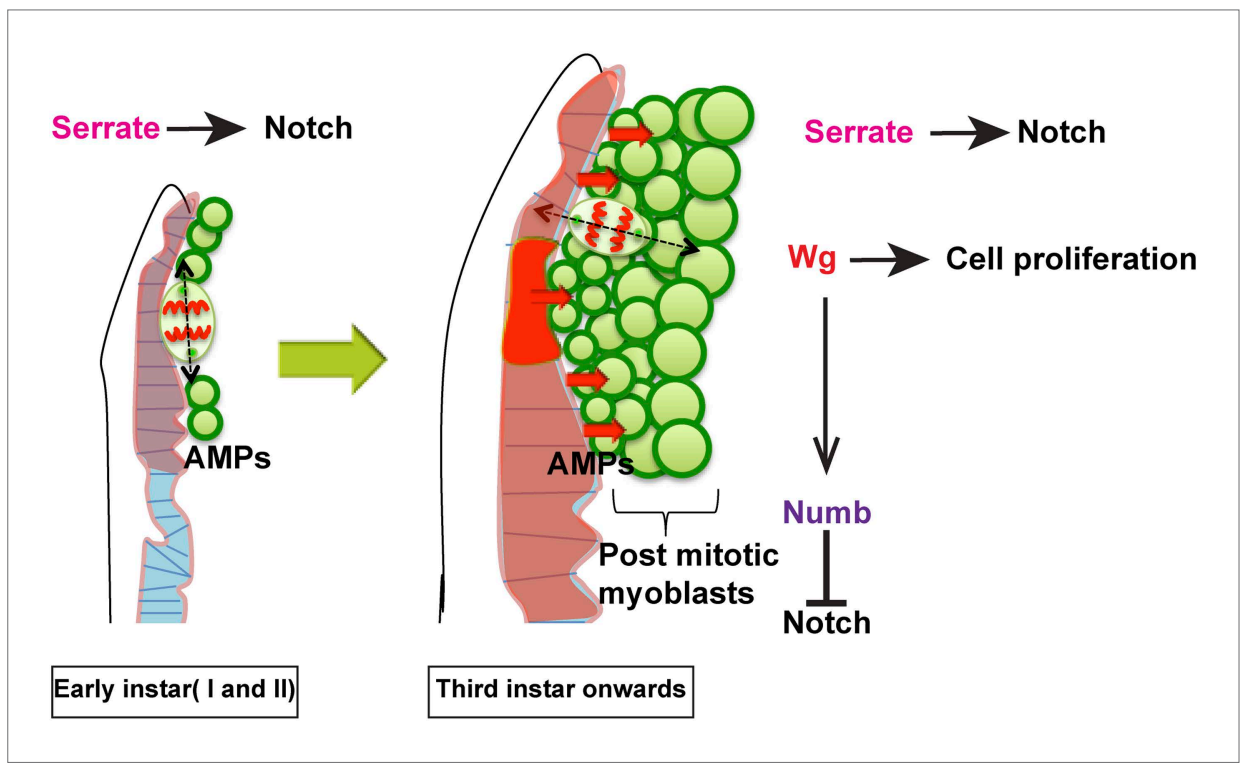

Figure 10. Model proposed. In the early instars (I and II) AMPs exhibit symmetric division along epithelium and Serrate-Notch signaling plays major role at this stage. In the third instar onwards the axis of cell division in AMPs changes to orthogonal orientation and expression of Wg in disc epithelium along with Serrate-Notch signaling regulates AMP proliferation. Wg signaling potentially regulates Numb, which inhibits Notch leading to asymmetric divisions. DOI: 10.7554/eLife.03126.019

muscle satellite cells are 'unfused' stem cells kept aside during early development. We also speculate that the novel stem cells that we observe could be satellite cells of flight muscle, used for repair upon muscle damage in a manner similar to that seen in vertebrates.

\section{Materials and methods}

\section{Fly strains and genetics}

Fly stocks were obtained from the Bloomington Drosophila Stock Centre (Indiana, USA) and, unless otherwise stated, were grown on standard cornmeal medium at $25^{\circ} \mathrm{C}$. The following transgenic lines were used.

For wild type Twin-spot MARCM experiments males of genotype +; FRT 40 A, UAS mCD8::GFP, UAS rCD2 RNAi; Dmef2-Gal4 were crossed to females Hsflp/Hsflp; FRT 40A, UAS rCD2::RFP, UAS GFP RNAi.

For Notch over expression and Numb downregulation twinspot MARCM experiments males of genotype +; FRT 40, UAS mCD8::GFP, UAS rCD2 RNAi; Dmef2-Gal4 were crossed to females of genotype Hsflp/Hsflp; FRT 40A, UAS rCD2::RFP, UAS GFP RNAi/Cyo Act-GFP; UAS NICD/TM6 or Hsflp/ Hsflp; FRT 40A, UAS rCD2::RFP, UAS GFP RNAi/Cyo Act-GFP; UAS Numb RNAi/TM6 Tb respectively.

For MARCM experiments females of genotype Hsflp/Hsflp; FRT 42 B, Tub Gal80 were crossed to males of genotype +; FRT 42 B UAS mCD8::GFP/Cyo Act-GFP; Dmef2-Gal4.

In knockdown and overexpression experiments:

+; +; Dmef2-Gal4, Gal80ts. UAS Notch RNAi (Bloom, 35213). UAS Numb RNAi (Bloom, 35045). UAS Wg RNAi (13352; VDRC, Austria). UAS Serrate RNAi (108348; VDRC, Austria). UAS NICD. UAS DN Notch. UAS DN TCF $\triangle N$. UAS (TCF) 24/Cyo GFP.

In mitotic spindle orientation experiments females of genotype + ; +; Dmef2-Gal4 were crossed to males of genotype P\{UASp-GFP-Cnn1\}26-1, w1118.

\section{Other stocks}

Serrate lacZ9.1 (Bachmann and Knust, 1998). Wg Sp-1/Cyo GFP. Wg Wotch $^{\text {ts }}$. wg $\{K O, N r t-W g\}$ (Alexandre et al., 2014).

\section{Immunohistochemistry and microscopy}

Wing discs were dissected from first instar (24-30 hr AEL), second instar (48-55 hr AEL) and third instar larval (72 hr onwards) stages and then fixed in $4 \%$ paraformaldehyde diluted in Phosphate 
buffered saline (PBS pH-7.5). Immunostaining was performed according to Ghazi et al. (2000). In brief, samples were then subjected to two washes of $0.3 \%$ PTX (PBS $+0.3 \%$ Triton-X) and $0.3 \%$ PBTX (PBS + 0.3\% Triton-X $+0.1 \% B S A$ ) for $15 \mathrm{~m}$ each. Primary antibody staining was performed for overnight at $4{ }^{\circ} \mathrm{C}$ on shaker and secondary antibodies were added following four washes of $0.3 \%$ PTX. Excess of unbound secondary antibodies were removed by two washes of $0.3 \%$ PTX following which samples were mounted in Vectashield mounting media. For immunostaining Anti Wg (Mouse, 1:100, DSHB), Anti-Twist (Rabbit, 1:100, kindly provided by S Roth, University of Cologne), Anti-NICD (Notch intracellular C-terminal domain) (Mouse, 1:100, DSHB), Anti-Numb (Rabbit, 1:100, kindly provided by Juergen Knoblich, IMBA, Vienna), Anti-GFP (Chick, 1:500, Abcam, Cambridge, UK), Anti-CD2 (Mouse, 1:100; Serotec, Raleigh, NC, USA), TO-PRO-3-lodide (1:1000, Invitrogen), Anti-DCAD2 (Rat, 1:200, DSHB), Anti-Beta Gal (Mouse, 1:50, DSHB), AntiPhospho histone 3 (Rabbit, 1:100, Millipore). Secondary antibodies (1:500) from Invitrogen conjugated with Alexa fluor-488, 568 and 647 were used in all staining procedures. Olympus FV 1000 confocal point scanning microscope was used for image acquisition, which were processed using ImageJ software.

Quantification of division axis was essentially performed as explained in the Egger et al. (2007).

\section{Twin-spot MARCM and MARCM experiments}

To generate twin-spot MARCM clones, a single heat shock of $15 \mathrm{~m}$ at $37^{\circ} \mathrm{C}$ was given to specifically staged larvae and then larvae were dissected and wing discs were removed. The samples were then processed for antibody staining as mentioned earlier. ImageJ software was used to determine the number of cells in each clone (Rasband WS, ImageJ U S. National Institutes of Health, Bethesda, Maryland, USA, http://imagej.nih.gov/ij/, 1997-2012).

\section{Edu labeling experiments}

For Edu labeling, larvae were aged for 24,48 and $72 \mathrm{hr}$ after hatching, on standard cornmeal media and then pulse labeled for $5 \mathrm{hr}$ on Edu ( $0.2 \mathrm{mM}$ final concentration) mixed cornmeal media (Daul et al., 2010). Half of the larvae from the $72 \mathrm{hr}$ stage were separated, dissected and processed for immunolabeling in the 'no chase' cohort. Remaining larvae were transferred to standard cornmeal media without Edu and allowed to develop until wandering third instar stage. Wing discs were then dissected and processed for immunolabeling studies. In both cohorts, Edu detection was performed according to the Click-iT Edu labeling kit (Invitrogen).

\section{Acknowledgements}

This work was possible due to the generous support from the Tata Institute of Fundamental Research and the National Centre for Biological Sciences. We thank the Centre for Nanotechnology, NCBS (Department of Science and Technology Grant No. SR/S5/NM- 36/2005), for the Olympus FV1000 microscopes in the Central Imaging and Flow facilities (NCBS). Major additional support came from grants from the Department of Biotechnology, the Department of Science and Technology, Government of India and the Swiss SNF. We thank the Drosophila community for generous supply of fly strains and antibodies. Special thanks to Juergen Knoblich and colleagues (IMBA, Austria) for the anti-Numb antibody.

\section{Additional information}

Competing interests

KVR: Senior editor, eLife. The other authors declare that no competing interests exist.

Funding

\begin{tabular}{ll} 
Funder & Author \\
\hline Department of Science and & Rajesh D Gunage, \\
Technology, Ministry of Science & K VijayRaghavan \\
and Technology &
\end{tabular}

Swiss National Science Foundation Heinrich Reichert

The funders had no role in study design, data collection and

interpretation, or the decision to submit the work for publication. 
Author contributions

RDG, Conception and design, Acquisition of data, Analysis and interpretation of data, Drafting or revising the article; HR, KVR, Conception and design, Analysis and interpretation of data, Drafting or revising the article

\section{References}

Alexandre C, Baena-Lopez A, Vincent JP. 2014. Patterning and growth control by membrane-tethered wingless. Nature 505:180-185. doi: 10.1038/nature12879.

Anant S, Roy S, VijayRaghavan K. 1998. Twist and Notch negatively regulate adult muscle differentiation in Drosophila. Development 1369:1361-1369.

Bachmann A, Knust E. 1998. Dissection of cis-regulatory elements of the Drosophila gene Serrate. Development Genes and Evolution 208:346-351. doi: 10.1007/s004270050190.

Barker N, van Es JH, Kuipers J, Kujala P, van den Born M, Cozijnsen M, Clevers H. 2007. Identification of stem cells in small intestine and colon by marker gene Lgr5. Nature 449:1003-1007. doi: 10.1038/nature06196.

Bate M, Rushton E, Currie DA. 1991. Cells with persistent twist expression are the embryonic precursors of adult muscles in Drosophila. Development 89:79-89.

Borrell V, Reillo I. 2012. Emerging roles of neural stem cells in cerebral cortex development and evolution. Developmental Neurobiology 72:955-971. doi: 10.1002/dneu.22013.

Brack AS, Conboy IM, Conboy MJ, Shen J, Rando TA. 2008. A temporal switch from notch to Wnt signaling in muscle stem cells is necessary for normal adult myogenesis. Cell Stem Cell 2:50-59. doi: 10.1016/j. stem.2007.10.006.

Brack AS, Rando TA. 2012. Tissue-specific stem cells: Lessons from the skeletal muscle satellite cell. Cell Stem Cell 10:504-514. doi: 10.1016/j.stem.2012.04.001.

Brand AH, Livesey FJ. 2011. Neural Stem Cell Biology in Vertebrates and Invertebrates: More Alike than Different? Neuron 70:719-729. doi: 10.1016/j.neuron.2011.05.016.

Brower DL, Lawrence PA, Wilcox M. 1981. Clonal analysis of the undifferentiated wing disk of Drosophila. Developmental Biology 86:448-455. doi: 10.1016/0012-1606(81)90203-7.

Bryson-Richardson RJ, Currie PD. 2008. The genetics of vertebrate myogenesis. Nature Reviews Genetics 9:632-646. doi: 10.1038/nrg2369.

Campos-Ortega JA. 1997. Asymmetic division: dynastic intricacies of neuroblast division. Current Biology 7:R726-R728. doi: 10.1016/S0960-9822(06)00367-8.

Carmena A, Murugasu-Oei B, Menon D, Jiménez F, Chia W. 1998. Inscuteable and numb mediate asymmetric muscle progenitor cell divisions during Drosophila myogenesis. Genes \& Development 12:304-315. doi: $10.1101 / \mathrm{gad} .12 .3 .304$.

Chai R, Kuo B, Wang T, Liaw EJ, Xia A, Jan TA, Liu Z, Taketo MM, Oghalai JS, Nusse R, Zuo J, Cheng AG. 2012. Wnt signaling induces proliferation of sensory precursors in the postnatal mouse cochlea. Proceedings of the National Academy of Sciences of USA 109:8167-8172. doi: 10.1073/pnas.1202774109.

Chen T, Heller E, Beronja S, Oshimori N, Stokes N, Fuchs E. 2012. An RNA interference screen uncovers a new molecule in stem cell self-renewal and long-term regeneration. Nature 485:104-108. doi: 10.1038/ nature10940.

Cohen B, Simcox AA, Cohen SM. 1993. Allocation of the thoracic imaginal primordia in the Drosophila embryo. Development 117:597-608.

Cooper RN, Tajbakhsh S, Mouly V, Cossu G, Buckingham M, Ura C. 1999. In vivo satellite cell activation via Myf5 and MyoD in regenerating mouse skeletal muscle. Journal of Cell Science 2901:2895-2901.

Cordero JB, Stefanatos RK, Scopelliti A, Vidal M, Sansom OJ. 2012. Inducible progenitor-derived wingless regulates adult midgut regeneration in Drosophila. The EMBO Journal 31:3901-3917. doi: 10.1038/emboj.2012.248.

Couturier L, Vodovar N, Schweisguth F. 2012. Endocytosis by Numb breaks Notch symmetry at cytokinesis. Nature Cell Biology 14:131-139. doi: 10.1038/ncb2419.

Crossley AC. 1972. Ultrastructural changes during transition of larval to adult intersegmental muscle at metamorphosis in the blowfly Calliphora erythrocephala. II. The formation of adult muscle. Journal of Embryology and Experimental Morphology 27:75-101.

Currie DA, Bate M. 1991. The development of adult abdominal muscles in Drosophila: myoblasts express twist and are associated with nerves. Development 113:91-102.

Daul AL, Komori H, Lee C-Y. 2010. EdU (5-ethynyl-2'-deoxyuridine) labeling of Drosophila mitotic neuroblasts. Cold Spring Harbor Protocols 2010:pdb.prot5461. doi: 10.1101/pdb.prot5461.

Doe CQ. 2008. Neural stem cells: balancing self-renewal with differentiation. Development 135:1575-1587. doi: $10.1242 /$ dev.014977.

Dutta D, Anant S, Ruiz-gomez M, Bate M, VijayRaghavan K. 2004. Founder myoblasts and fibre number during adult myogenesis in Drosophila. Development 131:3761-3772. doi: 10.1242/dev.01249.

Egger B, Boone JQ, Stevens NR, Brand AH, Doe CQ. 2007. Regulation of spindle orientation and neural stem cell fate in the Drosophila optic lobe. Neural Development 2:1. doi: 10.1186/1749-8104-2-1.

Egger B, Gold KS, Brand AH. 2010. Notch regulates the switch from symmetric to asymmetric neural stem cell division in the Drosophila optic lobe. Development 2987:2981-2987. doi: 10.1242/dev.051250.

Egger B, Gold KS, Brand AH. 2011. Regulating the balance between symmetric and asymmetric stem cell division in the developing brain. Fly 5:237-241. doi: 10.4161/fly.5.3.15640. 
Egger B, Chell JM, Brand AH. 2008. Insights into neural stem cell biology from flies. Philosophical Transactions of the Royal Society of London. Series B, Biological Sciences 363:39-56. doi: 10.1098/rstb.2006.2011.

Farkas LM, Huttner WB. 2008. The cell biology of neural stem and progenitor cells and its significance for their proliferation versus differentiation during mammalian brain development. Current Opinion in Cell Biology 20:707-715. doi: 10.1016/j.ceb.2008.09.008.

Fernandes J, Bate M, VijayRaghavan K. 1991. Development of the indirect flight muscles of Drosophila. Development 113:67-77.

Fernandes J, Celniker SE, Lewis EB, VijayRaghavan K. 1994. Muscle development in the four-winged Drosophila and the role of the Ultrabithorax gene. Current Biology 4:957-964. doi: 10.1016/S0960-9822(00)00219-0.

Frise E, Knoblich JA, Younger-Shepherd S, Jan LY, Jan YN. 1996. The Drosophila numb protein inhibits signaling of the Notch receptor during cell-cell interaction in sensory organ lineage. Proceedings of the National Academy of Sciences of USA 93:11925-11932. doi: 10.1073/pnas.93.21.11925.

Ghazi A, VijayRaghavan K. 2003. Muscle development in Drosophila. 702:691-702.

Ghazi A, Anant S, VijayRaghavan K. 2000. Apterous mediates development of direct flight muscles autonomously and indirect flight muscles through epidermal cues. Development 127:5309-5318.

Gilboa L, Lehmann R. 2006. Soma-germline interactions coordinate homeostasis and growth in the Drosophila gonad. Nature 443:97-100. doi: 10.1038/nature05068.

Gonzalez C. 2007. Spindle orientation, asymmetric division and tumour suppression in Drosophila stem cells. Nature Reviews Genetics 8:462-472. doi: 10.1038/nrg2103.

Greig S, Akam M. 1993. Homeotic genes autonomously specify one aspect of pattern in the Drosophila mesoderm. Nature 362:630-632. doi: 10.1038/362630a0.

Homem CC, Knoblich JA. 2012. Drosophila neuroblasts: a model for stem cell biology. Development 139:4297-4310. doi: 10.1242/dev.080515.

Jiang H, Edgar BA. 2009. EGFR signaling regulates the proliferation of Drosophila adult midgut progenitors. Development 136:483-493. doi: 10.1242/dev.026955.

Kassar-Duchossoy L, Giacone E, Gayraud-Morel B, Jory A, Gomès D, Tajbakhsh S. 2005. Pax3/Pax7 mark a novel population of primitive myogenic cells during development. Genes \& Development 19:1426-1431. doi: 10.1101/gad.345505.

Knoblich JA. 2001. Asymmetric cell division during animal development. Nature Reviews Molecular Cell Biology 2:11-20.

Konstantinides N, Averof M. 2014. A common cellular basis for muscle regeneration in arthropods and vertebrates. Science 343:788-791. doi: 10.1126/science.1243529.

Kriegstein A, Noctor S, Martínez-Cerdeño V. 2006. Patterns of neural stem and progenitor cell division may underlie evolutionary cortical expansion. Nature Reviews Neuroscience 7:883-890. doi: 10.1038/nrn2008.

Kubota K, Goto S, Eto K, Hayashi S. 2000. EGF receptor attenuates Dpp signaling and helps to distinguish the wing and leg cell fates in Drosophila. Development 127:3769-3776.

Lawrence PA. 1982. Cell lineage of the thoracic muscles of Drosophila. Cell 29:493-503.

Le Grand F, Rudnicki M. 2007. Satellite and stem cells in muscle growth and repair. Development 134:3953-3957. doi: $10.1242 /$ dev.005934.

Lopez-Garcia C, Klein AM, Simons BD, Winton DJ. 2010. Intestinal stem cell replacement follows a pattern of neutral drift. Science 330:822-825. doi: 10.1126/science.11962.

López-Onieva L, Fernández-Miñán A, González-Reyes A. 2008. Jak/Stat signalling in niche support cells regulates dpp transcription to control germline stem cell maintenance in the Drosophila ovary. Development 135:533-540. doi: 10.1242/dev.016121.

Losick VP, Morris LX, Fox DT, Spradling A. 2011. Drosophila stem cell niches: a decade of discovery suggests a unified view of stem cell regulation. Developmental Cell 21:159-171. doi: 10.1016/j. devcel.2011.06.018.

Mandal L, Martinez-Agosto JA, Evans CJ, Hartenstein V, Banerjee U. 2007. A Hedgehog- and Antennapediadependent niche maintains Drosophila haematopoietic precursors. Nature 446:320-324. doi: 10.1038/nature05585.

Martín FA, Morata G. 2006. Compartments and the control of growth in the Drosophila wing imaginal disc. Development 133:4421-4426. doi: 10.1242/dev.02618.

McGuire SE, Le PT, Osborn AJ, Matsumoto K, Davis RL. 2003. Spatiotemporal rescue of memory dysfunction in Drosophila. Science 302:1765-1768. doi: 10.1126/science.1089035.

Mathur D, Bost A, Driver I, Ohlstein B. 2010. A transient niche regulates the specification of Drosophila intestinal stem cells. Science 327:210-213. doi: 10.1126/science.1181958.

Maurange C, Cheng L, Gould AP. 2008. Temporal transcription factors and their targets schedule the end of neural proliferation in Drosophila. Cell 133:891-902. doi: 10.1016/j.cell.2008.03.034.

MAURO A. 1961. Satellite cell of skeletal muscle fibers. The Journal of Biophysical and Biochemical Cytology 9:493-495.

Megraw TL, Kilaru S, Turner FR, Kaufman TC. 2002. The centrosome is a dynamic structure that ejects PCM flares. Journal of Cell Science 115:4707-4718. doi: 10.1242/jcs.00134.

Micchelli CA, Perrimon N. 2006. Evidence that stem cells reside in the adult Drosophila midgut epithelium. Nature 439:475-479. doi: 10.1038/nature04371.

Morrison SJ, Kimble J. 2006. Asymmetric and symmetric stem-cell divisions in development and cancer. Nature 441:1068-1074. doi: 10.1038/nature04956.

Ngo KT, Wang J, Junker M, Kriz S, Vo G, Asem B, Olson JM, Banerjee U, Hartenstein V. 2010. Concomitant requirement for notch and Jak/Stat signaling during neuro-epithelial differentiation in the Drosophila optic lobe. Developmental Biology 346:284-295. doi: 10.1016/j.ydbio.2010.07.036. 
O'Brien LE, Soliman SS, Li X, Bilder D. 2011. Altered modes of stem cell division drive adaptive intestinal growth. Cell 147:603-614. doi: 10.1016/j.cell.2011.08.048.

Ohlstein B, Spradling A. 2007. Multipotent Drosophila intestinal stem cells specify daughter cell fates by differential notch signaling. Science 315:988-992. doi: 10.1126/science.1136606.

Orihara-Ono M, Toriya M, Nakao K, Okano H. 2011. Downregulation of Notch mediates the seamless transition of individual Drosophila neuroepithelial progenitors into optic medullar neuroblasts during prolonged G1. Developmental Biology 351:163-175. doi: 10.1016/j.ydbio.2010.12.044.

Phillips RG, Whittle JR. 1993. wingless expression mediates determination of peripheral nervous system elements in late stages of Drosophila wing disc development. Development 118:427-438.

Ranganayakulu G, Elliott DA, Harvey RP, Olson EN. 1998. Divergent roles for NK-2 class homeobox genes in cardiogenesis in flies and mice. Development 125:3037-3048.

Reddy BV, Rauskolb C, Irvine KD. 2010. Influence of fat-hippo and notch signaling on the proliferation and differentiation of Drosophila optic neuroepithelia. Development 137:2397-2408. doi: 10.1242/ dev.050013.

Reichert H. 2011. Cell Cycle in development. In: Kubiak JZ, editor. 53. p. 529-546. doi: 10.1007/9783-642-19065-0.

Reya T, Morrison SJ, Clarke MF, Weissman IL. 2001. Stem cells, cancer, and cancer stem cells. Nature 414:105-111. doi: $10.1038 / 35102167$.

Roy S, Shashidhara LS, VijayRaghavan K. 1997. Muscles in the Drosophila second thoracic segment are patterned independently of autonomous homeotic gene function. Current Biology 7:222-227. doi: 10.1016/ S0960-9822(06)00117-5.

Roy S, VijayRaghavan K. 1998. Patterning muscles using organizers: larval muscle templates and adult myoblasts actively interact to pattern the dorsal longitudinal flight muscles of Drosophila. The Journal of Cell Biology 141:1135-1145. doi: 10.1083/jcb.141.5.1135.

Roy S, VijayRaghavan K. 1997. Homeotic genes and the regulation of myoblast migration, fusion, and fibrespecific gene expression during adult myogenesis in Drosophila. Development 124:3333-3341.

Roy S, VijayRaghavan K. 1999. Muscle pattern diversification in Drosophila: the story of imaginal myogenesis. Bioessays 21:486-498. doi: 10.1002/(SICI)1521-1878(199906)21:6<486::AID-BIES5>3.0.CO;2-M.

Ruiz Gómez M, Bate M. 1997. Segregation of myogenic lineages in Drosophila requires numb. Development 124:4857-4866.

Sambasivan R, Tajbakhsh S. 2007. Skeletal muscle stem cell birth and properties. Seminars in Cell \& Developmental Biology 18:870-882. doi: 10.1016/j.semcdb.2007.09.013.

Sousa-Nunes R, Cheng LY, Gould AP. 2010. Regulating neural proliferation in the Drosophila CNS. Current Opinion in Neurobiology 20:50-57. doi: 10.1016/j.conb.2009.12.005.

Schnorrer F, Schönbauer C, Langer CCH, Dietzl G, Novatchkova M, Schernhuber K, Fellner M, Azaryan A, Radolf M, Stark A, Keleman K, Dickson BJ. 2010. Systematic genetic analysis of muscle morphogenesis and function in Drosophila. Nature 464:287-291. doi: 10.1038/nature08799.

Schönbauer C, Distler J, Jährling N, Radolf M, Dodt HU, Frasch M, Schnorrer F. 2011. Spalt mediates an evolutionarily conserved switch to fibrillar muscle fate in insects. Nature 479:406-409. doi: 10.1038/nature10559.

Sharma RP, Chopra VL. 1976. Effect of the wingless (wg1) mutation on wing and haltere development in Drosophila melanogaster. Developmental Biology 48:461-465. doi: 10.1016/0012-1606(76)90108-1.

Skeath JB, Doe CQ. 1998. Sanpodo and Notch act in opposition to numb to distinguish sibling neuron fates in the Drosophila CNS. Development 125:1857-1865.

Speicher SA, Thomas U, Hinz U, Knust E. 1994. The Serrate locus of Drosophila and its role in morphogenesis of the wing imaginal discs: control of cell proliferation. Development 120:535-544.

Sudarsan V, Anant S, Guptan P, VijayRaghavan K, Skaer H. 2001. Myoblast diversification and ectodermal signaling in Drosophila. Developmental Cell 1:829-839. doi: 10.1016/S1534-5807(01)00089-2.

Takashima S, Mkrtchyan M, Younossi-Hartenstein A, Merriam JR, Hartenstein V. 2008. The behaviour of Drosophila adult hindgut stem cells is controlled by Wnt and Hh signalling. Nature 454:651-655. doi: 10.1038/ nature07156.

Thisse B, Stoetzel C, Gorostiza-Thisse C, Perrin-Schmitt F. 1988. Sequence of the twist gene and nuclear localization of its protein in endomesodermal cells of early Drosophila embryos. The EMBO Journal 7:2175-2183.

Tomoyasu Y, Ueno N, Nakamura M. 2000. The decapentaplegic morphogen gradient regulates the notal wingless expression through induction of pannier and u-shaped in Drosophila. Mechanisms of Development 96:37-49. doi: 10.1016/S0925-4773(00)00374-9.

Tulina N, Matunis E. 2001. Control of stem cell self-renewal in Drosophila spermatogenesis by JAK-STAT signaling. Science 294:2546-2549. doi: 10.1126/science.1066700.

van Amerongen R, Nusse R. 2009. Towards an integrated view of Wnt signaling in development. Development 136:3205-3214. doi: 10.1242/dev.033910.

Vasyutina E, Lenhard DC, Birchmeier C. 2007. Notch function in myogenesis. Cell Cycle 6:1451-1454.

Wang L, Li Z, Cai Y. 2008. The JAK/STAT pathway positively regulates DPP signaling in the Drosophila germline stem cell niche. The Journal of Cell Biology 180:721-728. doi: 10.1083/jcb.200711022.

Wang YX, Rudnicki MA. 2011. Satellite cells, the engines of muscle repair. Nature Reviews Molecular Cell Biology 13:127-133. doi: 10.1038/nrm3265.

Williams JA, Bell JB, Carroll SB. 1991. Control of Drosophila wing and haltere development by the nuclear vestigial gene product. Genes \& Development 5:2481-2495. doi: 10.1101/gad.5.12b.2481. 
Yilmaz ÖH, Katajisto P, Lamming DW, Gültekin Y, Bauer-Rowe KE, Sengupta S, Birsoy K, Dursun A, Yilmaz VO, Selig M, Nielsen GP, Mino-Kenudson M, Zukerberg LR, Bhan AK, Deshpande V, Sabatini DM. 2012. mTORC1 in the Paneth cell niche couples intestinal stem-cell function to calorie intake. Nature 486:490-495. doi: 10.1038/ nature11163.

Yu HH, Chen CH, Shi L, Huang Y, Lee T. 2009. Twin-spot MARCM to reveal the developmental origin and identity of neurons. Nature Neuroscience 12:947-953. doi: 10.1038/nn.2345.

Zecca M, Basler K, Struhl G. 1996. Direct and long-range action of a wingless morphogen gradient. Cell 87:833-844. doi: 10.1016/S0092-8674(00)81991-1. 